\title{
KERNEL THEOREMS IN COORBIT THEORY
}

\author{
PETER BALAZS, KARLHEINZ GRÖCHENIG, AND MICHAEL SPECKBACHER
}

\begin{abstract}
We prove general kernel theorems for operators acting between coorbit spaces. These are Banach spaces associated to an integrable representation of a locally compact group and contain most of the usual function spaces (Besov spaces, modulation spaces, etc.). A kernel theorem describes the form of every bounded operator between a coorbit space of test functions and distributions by means of a kernel in a coorbit space associated to the tensor product representation. As special cases we recover Feichtinger's kernel theorem for modulation spaces and the recent generalizations by Cordero and Nicola. We also obtain a kernel theorem for operators between the Besov spaces $\dot{B}_{1,1}^{0}$ and $\dot{B}_{\infty, \infty}^{0}$.
\end{abstract}

\section{INTRODUCTION}

Kernel theorems assert that every "reasonable" operator can be written as a "generalized" integral operator. For instance, the Schwartz kernel theorem states that a continuous linear operator $A: \mathcal{S}\left(\mathbb{R}^{d}\right) \rightarrow \mathcal{S}^{\prime}\left(\mathbb{R}^{d}\right)$ possesses a unique distributional kernel $K \in \mathcal{S}^{\prime}\left(\mathbb{R}^{2 d}\right)$ such that

$$
\langle A f, g\rangle=\langle K, g \otimes f\rangle, \quad f, g \in \mathcal{S}\left(\mathbb{R}^{d}\right) .
$$

If $K$ is a locally integrable function, then

$$
\langle A f, g\rangle=\int_{\mathbb{R}^{d}} K(x, y) f(y) \overline{g(x)} d y d x, \quad f, g \in \mathcal{S}\left(\mathbb{R}^{d}\right),
$$

and thus $A$ has indeed the form of an integral operator. Similar kernel theorems hold for continuous operators from $\mathcal{D}\left(\mathbb{R}^{d}\right) \rightarrow \mathcal{D}^{\prime}\left(\mathbb{R}^{d}\right)$ [24, Theorem 5.2] and for Gelfand-Shilov spaces and their distribution spaces [21]. The importance of these kernel theorems stems from the fact that they offer a general formalism for the description of linear operators.

In the context of time-frequency analysis, Feichtinger's kernel theorem [12] (see also [18] and [23, Theorem 14.4.1]) states that every bounded linear operator from the modulation space $M^{1}\left(\mathbb{R}^{d}\right)$ to the modulation space $M^{\infty}\left(\mathbb{R}^{d}\right)$ can be represented in the form (11) with a kernel in $M^{\infty}\left(\mathbb{R}^{2 d}\right)$. The advantage of this kernel theorem is

Received by the editors March 27, 2019, and, in revised form, May 23, 2019

2010 Mathematics Subject Classification. Primary 42B35, 42C15, 46A32, 47B34.

Key words and phrases. Kernel theorems, coorbit theory, continuous frames, operator representation, tensor products, Hilbert-Schmidt operators.

The first and third authors were supported in part by the START-project FLAME ("Frames and Linear Operators for Acoustical Modeling and Parameter Estimation", Y 551-N13) of the Austrian Science Fund (FWF).

The second author was supported in part by the project P31887-N32 of the Austrian Science Fund (FWF).

(C)2019 by the authors under Creative Commons Attribution-Noncommercial 3.0 License (CC BY NC 3.0) 
that both the space of test functions $M^{1}\left(\mathbb{R}^{d}\right)$ and the distribution space $M^{\infty}\left(\mathbb{R}^{d}\right)=$ $M^{1}\left(\mathbb{R}^{d}\right)^{*}$ are Banach spaces and thus technically easier than the locally convex spaces $\mathcal{S}\left(\mathbb{R}^{d}\right)$ and $\mathcal{S}^{\prime}\left(\mathbb{R}^{d}\right)$.

Recently, Cordero and Nicola 8] revisited Feichtinger's kernel theorem and proved several new kernel theorems that "do not have a counterpart in distribution theory". They argue that "this reveals the superiority, in some respects, of the modulation space formalism upon distribution theory." While we agree fullheartedly with this claim, we would like to add a more abstract point of view and argue that the deeper reason for this superiority lies in the theory of coorbit spaces and in the convenience of Schur's test for integral operators. Indeed, we will prove kernel theorems similar to Feichtinger's kernel theorem for many coorbit spaces.

The main idea is to investigate operators in a transform domain after taking a short-time Fourier transform, a wavelet transform, or an abstract wavelet transform, i.e., a continuous transform with respect to a unitary group representation. In this new representation every operator between a suitable space of test functions and distributions is an integral operator. The standard boundedness conditions of Schur's test then yield strong kernel theorems.

The technical framework for this idea is coorbit theory, which was introduced and studied in [15, 17,22] for the construction and analysis of function spaces by means of a generalized wavelet transform. The main idea is that functions in the standard function spaces, such as Besov spaces and modulation spaces, can be characterized by the decay or integrability properties of an associated transform (the wavelet transform or the short-time Fourier transform). In the abstract setting, $G$ is a locally compact group and $\pi: G \rightarrow \mathcal{U}(\mathcal{H})$ is an irreducible, unitary, integrable representation of $G$. Leaving technical details aside, the coorbit space $\mathcal{C}_{o_{\pi}} L_{w}^{p}(G)$ consists of all distributions $f$ in a suitable distribution space such that the representation coefficient $g \mapsto\langle f, \pi(g) \psi\rangle$ is in the weighted space $L_{w}^{p}(G)$.

Next, let $G_{1}$ and $G_{2}$ be two locally compact groups, and let $\left(\pi_{1}, \mathcal{H}_{1}\right)$ and $\left(\pi_{2}, \mathcal{H}_{2}\right)$ be irreducible, unitary, integrable representations of $G_{1}$ and $G_{2}$, respectively.

Let $A$ be a bounded linear operator between $\mathcal{C} o_{\pi_{1}} L_{w_{1}}^{1}\left(G_{1}\right)$ and $\mathcal{C} o_{\pi_{2}} L_{1 / w_{2}}^{\infty}\left(G_{2}\right)$. Our main insight is that such an operator can be described by a kernel in a coorbit space that is related to the tensor product representation $\pi=\pi_{2} \otimes \pi_{1}$ of $G=G_{1} \times G_{2}$ on the tensor product space $\mathcal{H}_{2} \otimes \mathcal{H}_{1}$. The following non-technical formulation offers a flavor of our main result in Theorem 3 ,

A linear operator $A$ is bounded from $\mathcal{C}_{\pi_{1}} L_{w_{1}}^{1}\left(G_{1}\right)$ to $\mathcal{C}_{o_{\pi_{2}}} L_{1 / w_{2}}^{\infty}\left(G_{2}\right)$ if and only if there exists a kernel $K \in \mathcal{C}_{O_{\pi}} L_{w_{1}^{-1} \otimes w_{2}^{-1}}^{\infty}\left(G_{1} \times G_{2}\right)$ such that

$$
\langle A v, \varphi\rangle=\langle K, \varphi \otimes v\rangle
$$

for all $v \in \mathcal{C}_{o_{\pi_{1}}} L_{w_{1}}^{1}\left(G_{1}\right), \varphi \in \mathcal{C}_{o_{\pi_{2}}} L_{w_{2}}^{1}\left(G_{2}\right)$.

This statement is not just a mere abstraction and generalization of the classical kernel theorem. With the choice of a specific group and representation one obtains explicit kernel theorems. For instance, using the Schrödinger representation of the Heisenberg group, one recovers Feichtinger's original kernel theorem. The added value is our insight that the conditions on the kernel of [8] in terms of mixed modulation spaces [4] amount to coorbit spaces with respect to the tensor product representation. Choosing the $a x+b$-group and the continuous wavelet representation, one obtains a kernel theorem for all bounded operators between the Besov spaces $\dot{B}_{1,1}^{0}$ and $\dot{B}_{\infty, \infty}^{0}$ with a kernel in a space of dominating mixed smoothness. 
This class of function spaces has been studied extensively [31,32 and is by no means artificial.

By using suitable versions of Schur's test, it is then possible to derive characterizations for the boundedness of operators between other coorbit spaces. For example, in Theorem 7 we will prove the following, with $\frac{1}{p}+\frac{1}{q}=1$ :

(i) $A: \mathcal{C}_{\pi_{1}} L_{w_{1}}^{1}\left(G_{1}\right) \rightarrow \mathcal{C}_{\pi_{2}} L_{w_{2}}^{p}\left(G_{2}\right)$ bounded $\Leftrightarrow K \in \mathcal{C} o_{\pi} \mathcal{L}_{1 / w_{1} \otimes w_{2}}^{p, \infty}\left(G_{1} \times G_{2}\right)$,

(ii) $A: \mathcal{C}_{\pi_{1}} L_{w_{1}}^{p}\left(G_{1}\right) \rightarrow \mathcal{C}_{\pi_{\pi_{2}}} L_{w_{2}}^{\infty}\left(G_{2}\right)$ bounded $\Leftrightarrow K \in \mathcal{C} o_{\pi} L_{1 / w_{1} \otimes w_{2}}^{q, \infty}\left(G_{1} \times G_{2}\right)$,

where the mixed-norm Lebesgue spaces $\mathcal{L}^{p, q}$ and $L^{p, q}$ on $G_{1} \times G_{2}$ are defined in (23) and (24), respectively.

The paper is organized as follows. In Section 2 we present the basics of tensor products and coorbit space theory. The theory of coorbit spaces of kernels with respect to products of integrable representations is developed in Section 3 . Our main results, the kernel theorems, are proved in Section 4 and applied to particular examples of group representations in Section 5.

We note that our proofs require a meaningful formulation of coorbit theory. One can therefore prove kernel theorems also in the context of other coorbit space theories [6, 9], e.g., for certain reducible representations.

\section{Preliminaries on tensor Products AND COORBIT SPACES}

2.1. Tensor products and Hilbert-Schmidt operators. The theory of tensor products is at the heart of kernel theorems for operators. Algebraically, a simple tensor of two vectors (in two possibly different Hilbert spaces) is a formal product of two vectors $f_{1} \otimes f_{2}$, and the tensor product $\mathcal{H}_{1} \otimes \mathcal{H}_{2}$ is obtained by taking the completion of all linear combinations of simple tensors with respect to the inner product

$$
\left\langle f_{1} \otimes f_{2}, g_{1} \otimes g_{2}\right\rangle:=\left\langle f_{1}, g_{1}\right\rangle\left\langle g_{2}, f_{2}\right\rangle .
$$

This tensor product is homogeneous in the following sense: $\alpha \cdot\left(f_{1} \otimes f_{2}\right)=\left(\alpha f_{1}\right) \otimes$ $f_{2}=f_{1} \otimes\left(\bar{\alpha} f_{2}\right)$. Note explicitly that the product $f_{1} \otimes f_{2}$ is anti-linear in the second factor. In some books this is done by introducing the dual Hilbert space $\mathcal{H}_{2}^{\prime}$ 25].

If each Hilbert space is an $L^{2}$-space $\mathcal{H}_{1}=L^{2}(X, \mu), \mathcal{H}_{2}=L^{2}(Y, \nu)$, then the simple tensor $f \otimes g$ is just the product $(x, y) \mapsto f(x) \cdot \overline{g(y)}$, and the tensor product becomes the product space $\mathcal{H}_{1} \otimes \mathcal{H}_{2}=L^{2}(X, \mu) \otimes L^{2}(Y, \nu)=L^{2}(X \times Y, \mu \times \nu)$.

The connection between functions and operators arises in the analytic approach to tensor products. We interpret a function of two variables as an integral kernel for an operator. Thus a simple tensor $f_{1} \otimes f_{2}$ of two functions becomes the rank one operator $f \mapsto\left\langle f, f_{2}\right\rangle f_{1}$ with integral kernel $f_{1}(x) \overline{f_{2}(y)}$, and a general $k \in L^{2}(X \times Y, \mu \times \nu)$ becomes a Hilbert-Schmidt operator from $L^{2}(Y, \nu)$ to $L^{2}(X, \mu)$. The systematic, analytic treatment of general tensor products of two Hilbert spaces often defines the tensor product as a space of Hilbert-Schmidt operators between $\mathcal{H}_{2}$ and $\mathcal{H}_{1}$. We note that his definition is already based on the characterization of Hilbert-Schmidt operators and thus represents a non-trivial kernel theorem [7]. Whereas the working mathematician habitually identifies an operator with its distributional kernel, we will make the conceptual distinction between tensor products and operators for our study of kernel theorems.

In the sequel we will denote the (distributional) kernel of an integral operator by $k$ and the abstract kernel in a tensor product by $K$. 
2.2. Coorbit space theory. Let $G$ be a locally compact group with left Haar measure $\int_{G} \ldots d g$, let $\mathcal{H}$ be a separable Hilbert space, and let $\mathcal{U}(\mathcal{H})$ be the group of unitary operators acting on $\mathcal{H}$. A continuous unitary group representation $\pi: G \rightarrow$ $\mathcal{U}(\mathcal{H})$ is called square integrable [1,11] if it is irreducible and there exist $\psi \in \mathcal{H}$ such that

$$
\int_{G}|\langle\psi, \pi(g) \psi\rangle|^{2} d g<\infty .
$$

A non-zero vector $\psi$ satisfying (3) is called admissible. For every square integrable representation there exists a densely defined operator $T$ such that $\forall f_{1}, f_{2} \in$ $\mathcal{H}, \psi_{1}, \psi_{2} \in \operatorname{Dom}(T)$, one has

$$
\int_{G}\left\langle f_{1}, \pi(g) \psi_{1}\right\rangle\left\langle\pi(g) \psi_{2}, f_{2}\right\rangle d g=\left\langle T \psi_{2}, T \psi_{1}\right\rangle\left\langle f_{1}, f_{2}\right\rangle .
$$

For fixed $\psi_{1}=\psi_{2}=\psi$ the representation coefficient $f \mapsto V_{\psi} f(g):=\langle f, \pi(g) \psi\rangle$ is interpreted as a generalized wavelet transform. The orthogonality relation (4) then implies that $V_{\psi}$ is a multiple of an isometry from $\mathcal{H}$ to $L^{2}(G)$. By using a weak interpretation of vector-valued integrals, (4) can also be recast as the inversion formula

$$
f=\frac{1}{\|T \psi\|^{2}} \int_{G}\langle f, \pi(g) \psi\rangle \pi(g) \psi d g .
$$

For the rest of this paper we assume without loss of generality that the chosen admissible vectors $\psi$ are normalized, i.e., $\|T \psi\|=1$.

The adjoint operator $V_{\psi}^{*}: L^{2}(G) \rightarrow \mathcal{H}$ is formally defined by

$$
V_{\psi}^{*} F:=\int_{G} F(g) \pi(g) \psi d g .
$$

Other domains and convergence properties will be discussed later.

With this notation (5) says that $V_{\psi}^{*} V_{\psi}=I_{\mathcal{H}}$ for all admissible and normalized vectors $\psi$, which in the language of recent frame theory means that $\{\pi(g) \psi\}_{g \in G}$ is a continuous Parseval frame. By [5, Proposition 2.1] one can always assume that $G$ is $\sigma$-finite since we assume $\mathcal{H}$ to be separable.

In coorbit theory one needs much stronger hypotheses on $\pi$. The representation $\pi$ is called integrable with respect to a weight $w$ if there exists an admissible vector $\psi \in \mathcal{H}$ such that

$$
\int_{G}|\langle\psi, \pi(g) \psi\rangle| w(g) d g<\infty
$$

Let $g_{1}, g_{2}, g_{3} \in G$. We call a weight $w: G \rightarrow \mathbb{R}^{+}$submultiplicative if $w\left(g_{1} g_{2}\right) \leqslant$ $w\left(g_{1}\right) w\left(g_{2}\right)$ and a function $m: G \rightarrow \mathbb{R}^{+} w$-moderate if it satisfies $m\left(g_{1} g_{2} g_{3}\right) \leqslant$ $w\left(g_{1}\right) m\left(g_{2}\right) w\left(g_{3}\right)$. If $m$ is $w$-moderate, the weighted Lebesgue space $L_{m}^{p}(G)$ is then invariant under left translation $L_{x} f(y)=f\left(x^{-1} y\right)$ and under the right translation $R_{x} f(y)=f(y x)$. Throughout this paper, we will assume that the weight $w$ satisfies

$$
w(x) \geqslant C \max \left\{\alpha(x), \alpha\left(x^{-1}\right), \beta(x), \Delta\left(x^{-1}\right) \beta\left(x^{-1}\right)\right\},
$$

where $\alpha(x):=\left\|L_{x}\right\|_{L_{m}^{p}(G) \rightarrow L_{m}^{p}(G)}, \beta(x):=\left\|R_{x}\right\|_{L_{m}^{p}(G) \rightarrow L_{m}^{p}(G)}$, and $\Delta$ denotes the modular function of $G$. 
Our standing assumption is that the representation $\pi$ of $G$ possesses an admissible vector $\psi$ such that $V_{\psi} \psi \in L_{w}^{1}(G)$. We denote the corresponding set by

$$
\mathcal{A}_{w}(G):=\left\{\psi \in \mathcal{H}, \psi \neq 0: V_{\psi} \psi \in L_{w}^{1}(G)\right\} .
$$

For fixed $\psi \in \mathcal{A}_{w}(G)$ the linear version of $\mathcal{A}_{w}(G)$,

$$
\mathcal{H}_{w}^{1}:=\left\{f \in \mathcal{H}: V_{\psi} f \in L_{w}^{1}(G)\right\},
$$

is dense in $\mathcal{H}$. Let $\left(\mathcal{H}_{w}^{1}\right) \sim$ denote the anti-dual of $\mathcal{H}_{w}^{1}$, i.e., the space of anti-linear continuous functionals on $\mathcal{H}_{w}^{1}$. As $\mathcal{H}_{w}^{1}$ is dense in $\mathcal{H}$, it follows that the inner product on $\mathcal{H} \times \mathcal{H}$ extends to $\left(\mathcal{H}_{w}^{1}\right)^{\sim} \times \mathcal{H}_{w}^{1}$ and so does the generalized wavelet transform.

The coorbit space with respect to $L_{m}^{p}(G)$ is then defined by

$$
\mathcal{C} o_{\pi} L_{m}^{p}(G):=\left\{f \in\left(\mathcal{H}_{w}^{1}\right)^{\sim}: V_{\psi} f \in L_{m}^{p}(G)\right\}
$$

and is equipped with the natural norm

$$
\|f\|_{\mathcal{C}_{o_{\pi}} L_{m}^{p}(G)}:=\left\|V_{\psi} f\right\|_{L_{m}^{p}(G)} .
$$

With our assumptions on $\pi, \psi, m$, the coorbit space $\mathcal{C}_{\pi} L_{m}^{p}(G)$ is a Banach space [16. Alternatively, $\mathcal{C}_{\pi} L_{m}^{p}(G)$ for $p<\infty$ can be defined as the completion of $H_{w}^{1}$ with respect to this norm. Moreover,

(9) $\mathcal{C}_{o_{\pi}} L^{2}(G)=\mathcal{H}, \quad \mathcal{C}_{o_{\pi}} L_{w}^{1}(G)=\mathcal{H}_{w}^{1}, \quad$ and $\quad \mathcal{C}_{o_{\pi}} L_{1 / w}^{\infty}(G)=\left(\mathcal{H}_{w}^{1}\right)^{\sim}=\mathcal{H}_{1 / w}^{\infty}$, and

$$
\mathcal{H}_{w}^{1} \subseteq \mathcal{C}_{\pi} L_{m}^{p}(G) \subseteq \mathcal{H}_{1 / w}^{\infty}
$$

for $1 \leqslant p \leqslant \infty$ and $w$-moderate weight $m$. In the context of coorbit space theory the space $\mathcal{H}_{w}^{1}$ serves as a space of test functions, and $\mathcal{H}_{1 / w}^{\infty}$ is the corresponding distribution space.

We quickly recall some of the fundamental properties of coorbit spaces; see for example [16, Theorems 4.1 and 4.2 and Proposition 4.3].

Proposition 1. Let $\psi, \phi \in \mathcal{A}_{w}(G), f \in \mathcal{C}_{o_{\pi}} L_{m}^{p}(G), g \in \mathcal{C}_{o_{\pi}} L_{1 / m}^{q}(G)$, and $F \in$ $L_{m}^{p}(G)$. Then the following properties hold:

(i) $V_{\psi}: \mathcal{C}_{o_{\pi}} L_{m}^{p}(G) \rightarrow L_{m}^{p}(G)$ is an isometry.

(ii) $\mathcal{H}_{m}^{p}$ is invariant with respect to $\pi$ and

$$
\|\pi(g) f\|_{\mathcal{C}_{\pi} L_{m}^{p}(G)} \leqslant w(g)\|f\|_{\mathcal{C}_{\pi} L_{m}^{p}(G)} \text { for all } g \in G, f \in \mathcal{C}_{o_{\pi}} L_{m}^{p}(G) .
$$

(iii) $V_{\psi}^{*}: L_{m}^{p}(G) \rightarrow \mathcal{C}_{\pi} L_{m}^{p}(G)$ is continuous.

(iv) $V_{\psi}^{*} V_{\psi}=I_{\mathcal{C}_{\pi} L_{m}^{p}(G)}$.

(v) Correspondence principle: Let $F \in L_{m}^{p}(G)$. There exists $f \in \mathcal{C}_{o_{\pi}} L_{m}^{p}(G)$ such that $F=V_{\psi} f$ if and only if $F=F * V_{\psi} \psi$, where $*$ denotes convolution on $G$.

(vi) Duality: For $1 \leqslant p<\infty, \frac{1}{p}+\frac{1}{q}=1$, we have $\left(\mathcal{C}_{o_{\pi}} L_{m}^{p}(G)\right)^{*}=\mathcal{C}_{o_{\pi}} L_{1 / m}^{q}(G)$, where the duality is given by

$$
\langle f, g\rangle_{\mathcal{C}_{\pi} L_{m}^{p}(G), \mathcal{C}_{\pi} L_{m}^{q}(G)}=\left\langle V_{\psi} f, V_{\psi} g\right\rangle_{L_{m}^{p}(G), L_{1 / m}^{q}(G)} .
$$

(vii) The definition of $\mathcal{C}_{\pi} L_{m}^{p}(G)$ is independent of the particular choice of the window function from $\mathcal{A}_{w}(G)$. In particular, $\left\|V_{\psi} f\right\|_{L_{m}^{p}(G)} \asymp\left\|V_{\phi} f\right\|_{L_{m}^{p}(G)}$ for arbitrary non-zero $\phi, \psi \in \mathcal{A}_{w}(G)$. 
We furthermore need a result on the existence of atomic decompositions for the space $\mathcal{C}_{o_{\pi}} L_{w}^{1}(G)$; see [15, Theorem 4.7].

Theorem 2. Let $\psi \in \mathcal{A}_{w}(G)$. There exists a discrete subset $\left\{g_{i}\right\}_{i \in \mathcal{I}} \subset G$ and a collection of linear functionals $\lambda_{i}: \mathcal{C}_{\pi} L_{w}^{1}(G) \rightarrow \mathbb{C}, i \in \mathcal{I}$, such that

$$
f=\sum_{i \in \mathcal{I}} \lambda_{i}(f) \pi\left(g_{i}\right) \psi, \quad \text { with } \sum_{i \in \mathcal{I}}\left|\lambda_{i}(f)\right| w\left(g_{i}\right) \asymp\|f\|_{\mathcal{C}_{\pi} L_{w}^{1}(G)},
$$

and the sum converges absolutely in $\mathcal{C}_{\pi} L_{w}^{1}(G)$.

\section{FRAMES AND COORBIT SPACES VIA TENSOR PRODUCTS}

Let $G_{1}, G_{2}$ be two locally compact groups with unitary square integrable representations $\pi_{1}: G_{1} \rightarrow \mathcal{U}\left(\mathcal{H}_{1}\right)$ and $\pi_{2}: G_{2} \rightarrow \mathcal{U}\left(\mathcal{H}_{2}\right)$. For $g:=\left(g_{1}, g_{2}\right) \in G:=G_{1} \times G_{2}$ the tensor representation $\pi: G \rightarrow \mathcal{U}\left(\mathcal{H}_{2} \otimes \mathcal{H}_{1}\right)$,

$$
\pi(g):=\pi_{2}\left(g_{2}\right) \otimes \pi_{1}\left(g_{1}\right),
$$

acts on a simple tensor $\Psi:=\psi_{2} \otimes \psi_{1} \in \mathcal{H}_{2} \otimes \mathcal{H}_{1}$ by

$$
\pi(g)\left(\psi_{2} \otimes \psi_{1}\right)=\pi_{2}\left(g_{2}\right) \psi_{2} \otimes \pi_{1}\left(g_{1}\right) \psi_{1} .
$$

It follows immediately that $\pi$ is a unitary representation of $G$ on $\mathcal{H}_{2} \otimes \mathcal{H}_{1}$. Moreover, $\pi$ is irreducible (e.g., by [34, Section 4.4, Theorem 6]). Note that the order of indices is in agreement with the formulation of the kernel theorem in Theorem 3 .

If we interpret the simple tensor $\Psi=\psi_{2} \otimes \psi_{1}$ as the rank-one operator $f \mapsto$ $\psi_{1}(f) \psi_{2}$ with $\psi_{1} \in \mathcal{H}_{1}^{\prime}$, then we can write (12) as

$$
\pi(g)(\Psi)(f)=\left(\pi_{1}^{\prime}\left(g_{1}\right) \psi_{1}\right)(f) \cdot \pi_{2}\left(g_{2}\right) \psi_{2}=\left(\pi_{2}\left(g_{2}\right) \psi_{2} \otimes \pi_{1}^{\prime}\left(g_{1}\right) \psi_{1}\right)(f),
$$

where the contragredient representation $\pi_{1}^{\prime}: G_{1} \rightarrow G L\left(\mathcal{H}_{1}^{\prime}\right)$ of $\pi_{1}$ is defined as $\left(\pi_{1}^{\prime}\left(g_{1}\right) \psi_{1}\right)(f)=\psi_{1}\left(\pi_{1}\left(g_{1}^{-1}\right) f\right)$; see [34, Section 3.1].

In case we treat the tensor product as a space of Hilbert-Schmidt operators, $\pi$ acts on $A \in \mathcal{H S}\left(\mathcal{H}_{1}, \mathcal{H}_{2}\right)$ as

$$
\pi(g) A=\pi_{2}\left(g_{2}\right) A \pi_{1}\left(g_{1}\right)^{*} .
$$

The generalized wavelet transform of a simple tensor $f_{2} \otimes f_{1}$ with respect to a "wavelet" $\Psi=\psi_{2} \otimes \psi_{1}$ is given by

$$
\begin{aligned}
V_{\Psi}\left(f_{2} \otimes f_{1}\right)(g) & =\left\langle f_{2} \otimes f_{1},\left(\pi_{2}\left(g_{2}\right) \otimes \pi_{1}\left(g_{1}\right)\right)\left(\psi_{2} \otimes \psi_{1}\right)\right\rangle \\
& =\left\langle f_{2}, \pi_{2}\left(g_{2}\right) \psi_{2}\right\rangle \overline{\left\langle f_{1}, \pi_{1}\left(g_{1}\right) \psi_{2}\right\rangle} \\
& =V_{\psi_{2}} f_{2}\left(g_{2}\right) \overline{V_{\psi_{1}} f_{1}\left(g_{1}\right)} .
\end{aligned}
$$

Thus, the wavelet transform of the tensor product representation factors into the product of wavelet transforms on $G_{1}$ and $G_{2}$. Strictly speaking, we would have to write $V_{\psi_{i}}^{\pi_{i}} f_{i}$ to indicate the underlying representation, but we omit the reference to the group to keep notation simple.

Throughout this paper we consider only separable weights $w: G \rightarrow \mathbb{R}_{+}$with $w(g)=\left(w_{1} \otimes w_{2}\right)(g)=w_{1}\left(g_{1}\right) w_{2}\left(g_{2}\right)$ and $m(g)=\left(m_{1} \otimes m_{2}\right)(g)=m_{1}\left(g_{1}\right) m_{2}\left(g_{2}\right)$, where $w_{i}$ is submultiplicative and $m_{i}$ is $w_{i}$-moderate. Moreover we write $(1 / w)(g)=$ $\left(w_{1} \otimes w_{2}\right)(g)^{-1}$. It follows from (13) that the tensor representation $\pi_{2} \otimes \pi_{1}$ of two square-integrable representations is again square-integrable and that the tensor $\Psi=\psi_{2} \otimes \psi_{1}$ of two admissible vectors $\psi_{2}$ and $\psi_{1}$ is admissible for $\pi$. Likewise, if $w=w_{1} \otimes w_{2}$ and $\psi_{1} \in \mathcal{A}_{w_{1}}\left(G_{1}\right), \psi_{2} \in \mathcal{A}_{w_{2}}\left(G_{2}\right)$, then $\psi_{2} \otimes \psi_{1} \in \mathcal{A}_{w}\left(G_{1} \times G_{2}\right)$ 
(where we assume that $w_{i}, i=1,2$, satisfies (7)). Therefore all definitions and results of Section 2.2 hold for the representation $\pi=\pi_{2} \otimes \pi_{1}$ and $\Psi=\psi_{2} \otimes \psi_{1}$. In particular, the orthogonality relation (4), the inversion formula (5), Proposition 1 , and Theorem 2 hold for suitable admissible vectors $\Psi=\psi_{2} \otimes \psi_{1}$.

\section{KeRnel THEOREMS}

In this section we derive the general kernel theorems for operators between coorbit spaces. The basic idea comes from linear algebra, where a linear operator is identified with its matrix with respect to a basis. In coorbit theory the basic structure consists of the vectors $\pi(g) \psi$. Thus in analogy to linear algebra we try to describe an operator $A: \mathcal{H}_{1} \rightarrow \mathcal{H}_{2}$ by the kernel (= continuous matrix)

$$
k_{A}\left(g_{1}, g_{2}\right)=\left\langle A \pi_{1}\left(g_{1}\right) \psi_{1}, \pi_{2}\left(g_{2}\right) \psi_{2}\right\rangle .
$$

This can be seen as a continuous Galerkin-like representation of the operator $A$ [2,3]. The idea goes back to coherent state theory [30, Ch. 1.6]. One of its goals is to associate to every operator $A$ a function or symbol $k_{A}$, and (14) is one of the many possibilities to do so.

Assume that $A: \mathcal{C}_{\pi_{1}} L_{w_{1}}^{1}\left(G_{1}\right) \rightarrow \mathcal{C}_{\pi_{2}} L_{1 / w_{2}}^{\infty}\left(G_{2}\right)$ and $f \in \mathcal{C}_{\pi_{1}} L_{w_{1}}^{1}\left(G_{1}\right)$, i.e., $A$ maps "test functions" to "distributions". By using the inversion formula (5) for $f$ and applying $A$ to it, it follows formally that

$$
A f=\int_{G_{1}}\left\langle f, \pi_{1}\left(g_{1}\right) \psi_{1}\right\rangle A \pi_{1}\left(g_{1}\right) \psi_{1} d g_{1},
$$

and furthermore that

$$
\begin{aligned}
V_{\psi_{2}}(A f)\left(g_{2}\right) & =\left\langle A f, \pi_{2}\left(g_{2}\right) \psi_{2}\right\rangle=\int_{G_{1}}\left\langle f, \pi_{1}\left(g_{1}\right) \psi_{1}\right\rangle\left\langle A \pi_{1}\left(g_{1}\right) \psi_{1}, \pi_{2}\left(g_{2}\right) \psi_{2}\right\rangle d g_{1} \\
& =\int_{G}\left\langle f, \pi_{1}\left(g_{1}\right) \psi_{1}\right\rangle k_{A}\left(g_{1}, g_{2}\right) d g_{1} .
\end{aligned}
$$

Let

$$
\mathfrak{A} F\left(g_{2}\right)=\int_{G_{1}} F\left(g_{1}\right) k_{A}\left(g_{1}, g_{2}\right) d g_{1}
$$

be the integral operator with the kernel $k_{A}$. Then (15) can be written as

$$
V_{\psi_{2}} A f=\mathfrak{A} V_{\psi_{1}} f
$$

or, equivalently,

$$
A=V_{\psi_{2}}^{*} \mathfrak{A} V_{\psi_{1}}
$$

Using this factorization, the computation in (15) can be given a precise meaning on coorbit spaces. Identity (18) is the heart of the kernel theorems. The combination of the properties of the generalized wavelet transform (Proposition 10) and boundedness properties of integral operators yields powerful and very general kernel theorems. 
We will first show the existence of a generalized kernel for operators mapping the space of test functions $\mathcal{C}_{\pi_{1}} L_{w_{1}}^{1}\left(G_{1}\right)$ into the distribution space $\mathcal{C}_{\pi_{\pi_{2}}} L_{1 / w_{2}}^{\infty}\left(G_{2}\right)$. Subsequently, we will characterize continuous operators in certain subclasses.

Theorem 3. Let $G_{1}$ and $G_{2}$ be two locally compact groups, and let $\left(\pi_{j}, \mathcal{H}_{j}\right)$ be integrable, unitary, irreducible representations of $G_{j}$, such that $\mathcal{A}_{w_{j}}\left(G_{j}\right) \neq \emptyset$ for $j=1,2$.

(i) Every kernel $K \in \mathcal{C}_{\pi} L_{1 / w}^{\infty}\left(G_{1} \times G_{2}\right)$ defines a unique linear operator $A$ : $\mathcal{C}_{\pi_{\pi_{1}}} L_{w_{1}}^{1}\left(G_{1}\right) \rightarrow \mathcal{C}_{O_{\pi_{2}}} L_{1 / w_{2}}^{\infty}\left(G_{2}\right)$ by means of

$$
\langle A v, \varphi\rangle=\langle K, \varphi \otimes v\rangle
$$

for all $v \in \mathcal{C}_{o_{\pi_{1}}} L_{w_{1}}^{1}\left(G_{1}\right)$ and $\varphi \in \mathcal{C}_{o_{\pi_{2}}} L_{w_{2}}^{1}\left(G_{2}\right)$. The operator norm satisfies

$$
\|A\|_{O p} \asymp\|K\|_{\mathcal{C}_{\pi} L_{1 / w}^{\infty}}(G)
$$

and

$$
k_{A}=V_{\Psi} K
$$

(ii) Kernel theorem: Conversely, if $A: \mathcal{C}_{\pi_{1}} L_{w_{1}}^{1}\left(G_{1}\right) \rightarrow \mathcal{C}_{\pi_{\pi_{2}}} L_{1 / w_{2}}^{\infty}\left(G_{2}\right)$ is bounded, then there exists a unique kernel $K \in \mathcal{C}_{o_{\pi}} L_{1 / w}^{\infty}\left(G_{1} \times G_{2}\right)$ such that (19) holds.

Proof. (i) Fix $K \in \mathcal{C}_{o_{\pi}} L_{1 / w}^{\infty}(G)$ with $G=G_{1} \times G_{2}$, and let $v \in \mathcal{C}_{\pi_{1}} L_{w_{1}}^{1}\left(G_{1}\right), \varphi \in$ $\mathcal{C}_{o_{\pi_{2}}} L_{w_{2}}^{1}\left(G_{2}\right)$ be arbitrary. By (13) it follows that $\varphi \otimes v \in \mathcal{C} o_{\pi} L_{w}^{1}(G)$. Therefore, the duality in (19) is well-defined and

$$
\begin{aligned}
|\langle K, \varphi \otimes v\rangle| & \leqslant\|K\|_{\mathcal{C}_{o_{\pi} L_{1 / w}}^{\infty}(G)}\|\varphi \otimes v\|_{\mathcal{C}_{o_{\pi} L_{w}^{1}}^{1}(G)} \\
& =\|K\|_{\mathcal{C}_{o_{\pi}} L_{1 / w}^{\infty}(G)}\|\varphi\|_{\mathcal{C}_{o_{2}} L_{w_{2}}^{1}\left(G_{2}\right)}\|v\|_{\mathcal{C}_{o_{\pi_{1}} L_{w_{1}}^{1}}\left(G_{1}\right)} .
\end{aligned}
$$

Therefore, if we fix $v$, the mapping $\varphi \mapsto\langle K, \varphi \otimes v\rangle$ is a bounded, anti-linear functional on $\mathcal{C}_{\pi_{2}} L_{w_{2}}^{1}\left(G_{2}\right)$, which we call $A v \in \mathcal{C}_{o_{\pi_{2}}} L_{1 / w_{2}}^{\infty}\left(G_{2}\right)$. The map $v \mapsto A v$ is clearly linear, and (19) defines a linear operator $A: \mathcal{C}_{\pi_{1}} L_{w_{1}}^{1}\left(G_{1}\right) \rightarrow \mathcal{C}_{o_{\pi_{2}}} L_{1 / w_{2}}^{\infty}\left(G_{2}\right)$. The estimate (22) implies that

$$
\|A v\|_{\mathcal{C}_{\pi_{2}} L_{1 / w_{2}}^{\infty}\left(G_{2}\right)} \leqslant\|K\|_{\mathcal{C}_{o_{\pi}} L_{1 / w}^{\infty}(G)}\|v\|_{\mathcal{C}_{o_{\pi_{1}} L_{w_{1}}^{1}}^{1}\left(G_{1}\right)},
$$

and thus

$$
\|A\|_{\mathrm{Op}} \leqslant\|K\|_{\mathcal{C}_{\pi} L_{1 / w}^{\infty}(G)}
$$

(ii) To prove the converse, we need to show that the mapping $K \mapsto A$ is one-toone and onto.

Uniqueness: Let us assume that the kernel $\mathcal{K} \in \mathcal{C}_{o_{\pi}} L_{1 / w}^{\infty}(G)$ also satisfies

$$
\langle A v, \varphi\rangle=\langle K, \varphi \otimes v\rangle=\langle\mathcal{K}, \varphi \otimes v\rangle
$$


for every $v \in \mathcal{C}_{\pi_{1}} L_{w_{1}}^{1}\left(G_{1}\right), \varphi \in \mathcal{C}_{\pi_{2}} L_{w_{2}}^{1}\left(G_{2}\right)$. By Theorem 2, there exists a discrete set $\left\{\gamma_{i}\right\}_{i \in \mathcal{I}} \subset G$ such that every $F \in \mathcal{C}_{o_{\pi}} L_{w}^{1}(G)$ can be written as

$$
F=\sum_{i \in \mathcal{I}} \lambda_{i}(F) \pi\left(\gamma_{i}\right)\left(\psi_{2} \otimes \psi_{1}\right)
$$

with unconditional convergence in $\mathcal{C}_{o_{\pi}} L_{w}^{1}(G)$ and

$$
\sum_{i}\left|\lambda_{i}(F)\right| w\left(\gamma_{i}\right) \leqslant C\|F\|_{\mathcal{C}_{\pi} L_{w}^{1}\left(G_{1} \times G_{2}\right)} .
$$

Since $\pi\left(\gamma_{i}\right)\left(\psi_{2} \otimes \psi_{1}\right)=\pi\left(\gamma_{i, 2}\right) \psi_{2} \otimes \pi\left(\gamma_{i, 1}\right) \psi_{1}$, we conclude that

$$
\begin{aligned}
\langle K, F\rangle & =\sum_{i \in \mathcal{I}} \overline{\lambda_{i}(F)}\left\langle K, \pi\left(\gamma_{i, 2}\right) \psi_{2} \otimes \pi\left(\gamma_{i, 1}\right) \psi_{1}\right\rangle \\
& =\sum_{i \in \mathcal{I}} \overline{\lambda_{i}(F)}\left\langle\mathcal{K}, \pi\left(\gamma_{i, 2}\right) \psi_{2} \otimes \pi\left(\gamma_{i, 1}\right) \psi_{1}\right\rangle \\
& =\langle\mathcal{K}, F\rangle .
\end{aligned}
$$

As this equality holds for every $F \in \mathcal{C}_{o_{\pi}} L_{w}^{1}(G)$, it follows that $K=\mathcal{K}$.

Surjectivity: Let us assume that $A: \mathcal{C}_{\pi_{1}} L_{w_{1}}^{1}\left(G_{1}\right) \rightarrow \mathcal{C}_{\pi_{2}} L_{1 / w_{2}}^{\infty}\left(G_{2}\right)$ is bounded. Then the kernel $k_{A}$ defined in (14) is an element of $L_{1 / w}^{\infty}\left(G_{1} \times G_{2}\right)$, because

$$
\begin{aligned}
\left|k_{A}(g)\right| & =\left|\left\langle A \pi_{1}\left(g_{1}\right) \phi, \pi_{2}\left(g_{2}\right) \psi\right\rangle\right| \\
& \leqslant\|A\|_{O p}\left\|\pi_{1}\left(g_{1}\right) \phi\right\|_{\mathcal{C}_{\pi_{1}} L_{w_{1}}^{1}\left(G_{1}\right)}\left\|\pi_{2}\left(g_{2}\right) \psi\right\|_{\mathcal{C}_{o_{2}} L_{w_{2}}^{1}\left(G_{2}\right)} \\
& \leqslant\|A\|_{O p} w_{1}\left(g_{1}\right)\|\phi\|_{\mathcal{C}_{\pi_{1}} L_{w_{1}}^{1}\left(G_{1}\right)} w_{2}\left(g_{2}\right)\|\psi\|_{\mathcal{C}_{o_{2}} L_{w_{2}}^{1}}^{1}\left(G_{2}\right)
\end{aligned}
$$

We claim that $k_{A}$ is a generalized wavelet transform. Precisely, there exists $K \in$ $\mathcal{C}_{o_{\pi}} L_{1 / w}^{\infty}\left(G_{1} \times G_{2}\right)$ such that $k_{A}=V_{\Psi} K$. To prove this claim, we use Proposition 1(v), which asserts that $k_{A}=V_{\psi} K$ for some $K \in \mathcal{C}_{\pi} L_{1 / w}^{\infty}(G)$ if and only if $k_{A}=k_{A} * V_{\Psi} \Psi$.

As $k_{A} \cdot V_{\Psi}(\pi(g) \Psi) \in L^{1}\left(G_{1} \times G_{2}\right)$, we may choose the most convenient order of integration and apply the reproducing formula of Proposition $1(\mathbf{v})$ consecutively to the representations $\pi_{1}$ and $\pi_{2}$. Using (13) we obtain

$$
\begin{aligned}
\left(k_{A} * V_{\Psi} \Psi\right)(g) & =\int_{G} k_{A}(h) V_{\Psi} \Psi\left(h^{-1} g\right) d h \\
& =\int_{G_{1}} \int_{G_{2}} V_{\psi_{2}}\left(A \pi_{1}\left(h_{1}\right) \psi_{1}\right)\left(h_{2}\right) V_{\psi_{2}} \psi_{2}\left(h_{2}^{-1} g_{2}\right) d h_{2} \overline{V_{\psi_{1}} \psi_{1}\left(h_{1}^{-1} g_{1}\right)} d h_{1} \\
& =\int_{G_{1}}\left(V_{\psi_{2}}\left(A \pi_{1}\left(h_{1}\right) \psi_{1}\right) * V_{\psi_{2}} \psi_{2}\right)\left(g_{2}\right) \overline{V_{\psi_{1}} \psi_{1}\left(h_{1}^{-1} g_{1}\right)} d h_{1} \\
& =\int_{G_{1}}\left\langle A \pi_{1}\left(h_{1}\right) \psi_{1}, \pi_{2}\left(g_{2}\right) \psi_{2}\right\rangle \overline{V_{\psi_{1}} \psi_{1}\left(h_{1}^{-1} g_{1}\right)} d h_{1}=(*) .
\end{aligned}
$$

At this point we note that by the assumption on $A$ there exists a unique operator $A^{\prime}: \mathcal{C} o_{\pi_{2}} L_{w_{2}}^{1}\left(G_{2}\right) \rightarrow \mathcal{C} o_{\pi_{1}} L_{1 / w_{1}}^{\infty}\left(G_{1}\right)$ that satisfies

$$
\langle A v, \varphi\rangle=\left\langle v, A^{\prime} \varphi\right\rangle
$$


for every $v \in \mathcal{C}_{o_{\pi_{1}}} L_{w_{1}}^{1}\left(G_{1}\right)$ and $\varphi \in \mathcal{C}_{o_{\pi_{2}}} L_{w_{2}}^{1}\left(G_{2}\right)$. By its definition, $A^{\prime}$ is weak*continuous. We continue with the integration over $G_{1}$ and obtain

$$
\begin{aligned}
(*) & =\overline{\int_{G_{1}}\left\langle A^{\prime} \pi_{2}\left(g_{2}\right) \psi_{2}, \pi_{1}\left(h_{1}\right) \psi_{1}\right\rangle V_{\psi_{1}} \psi_{1}\left(h_{1}^{-1} g_{1}\right) d h_{1}} \\
& =\overline{\left(V_{\psi_{1}}\left(A^{\prime} \pi_{2}\left(g_{2}\right) \psi_{2}\right) * V_{\psi_{1}} \psi_{1}\right)\left(g_{1}\right)}=\overline{\left\langle A^{\prime} \pi_{2}\left(g_{2}\right) \psi_{2}, \pi_{1}\left(g_{1}\right) \psi_{1}\right\rangle} \\
& =\left\langle A \pi_{1}\left(g_{1}\right) \psi_{1}, \pi_{2}\left(g_{2}\right) \psi_{2}\right\rangle=k_{A}(g) .
\end{aligned}
$$

By Proposition 1( $(\bar{v})$ there exists a kernel $K \in \mathcal{C}_{o_{\pi}} L_{1 / w}^{\infty}\left(G_{1} \times G_{2}\right)$ such that $k_{A}\left(g_{1}, g_{2}\right)=V_{\Psi} K\left(g_{1}, g_{2}\right)$. By the first part of the proof $K$ defines an operator $B: \mathcal{C}_{\pi_{\pi_{1}}} L_{w_{1}}^{1}\left(G_{1}\right) \rightarrow \mathcal{C}_{o_{\pi_{2}}} L_{1 / w_{2}}^{\infty}\left(G_{2}\right)$ by means of $\langle B v, \phi\rangle=\langle K, \phi \otimes v\rangle$. In particular,

$$
\begin{aligned}
\left\langle B \pi_{1}\left(g_{1}\right) \psi_{1}, \pi_{2}\left(g_{2}\right) \psi_{2}\right\rangle & =\left\langle K, \pi_{2}\left(g_{2}\right) \psi_{2} \otimes \pi_{1}\left(g_{1}\right) \psi_{1}\right\rangle=V_{\Psi} K\left(g_{1}, g_{2}\right) \\
& =k_{A}\left(g_{1}, g_{2}\right)=\left\langle A \pi_{1}\left(g_{1}\right) \psi_{1}, \pi_{2}\left(g_{2}\right) \psi_{2}\right\rangle .
\end{aligned}
$$

Consequently, $B \pi_{1}\left(g_{1}\right) \psi_{1}=A \pi_{1}\left(g_{1}\right) \psi_{1}$ for all $g_{1} \in G_{1}$. This identity extends to all finite linear combinations of vectors $\pi_{1}\left(g_{1}\right) \psi_{1}$ and by Theorem 2 to $\mathcal{C}_{o_{\pi_{1}}} L_{w_{1}}^{1}\left(G_{1}\right)$. Thus $B=A$, and we have shown that the map from kernels to operators is onto.

The map $K \mapsto A$ is bounded and invertible. By the inverse mapping theorem we obtain that $\|K\|_{\mathcal{C}_{\pi} L_{1 / w}^{\infty}(G)} \leqslant C\|A\|_{\text {Op }}$, which proves (20).

Remark 4. It is crucial to interpret the brackets in (19) correctly. For utmost precision, we would have to write

$$
\langle A v, \varphi\rangle_{\mathcal{C}_{\pi_{2}} L_{1 / w_{2}}^{\infty}}\left(G_{2}\right), \mathcal{C}_{\pi_{2}} L_{w_{2}}^{1}\left(G_{2}\right)=\langle K, \varphi \otimes v\rangle_{\mathcal{C}_{\pi} L_{1 / w}^{\infty}(G), \mathcal{C} o_{\pi} L_{w}^{1}(G)},
$$

but we feel that this notation would distract from the analogy to distribution theory.

The injectivity of the mapping $K \mapsto A$ from kernels to operators is closely related to an important property of the coorbit spaces $\mathcal{C}_{\pi_{1}} L_{w_{1}}^{1}\left(G_{1}\right)$. This socalled tensor product property has gained considerable importance in certain special cases [13, Theorem $7 \mathrm{D}]$ and $[26$. We therefore state and prove a general version. Recall that the projective tensor product of two Banach spaces $B_{1}$ and $B_{2}$ is defined to be

$$
B_{1} \widehat{\otimes} B_{2}=\left\{f=\sum_{i \in \mathcal{I}} \phi_{i} \otimes \psi_{i}: \phi_{i} \in B_{1}, \psi_{i} \in B_{2} \quad \text { and } \quad \sum_{i \in \mathcal{I}}\left\|\phi_{i}\right\|_{B_{1}}\left\|\psi_{i}\right\|_{B_{2}}<\infty\right\} .
$$

The norm is given as $\|f\|_{\widehat{\otimes}}=\inf \sum_{i \in \mathcal{I}}\left\|\phi_{i}\right\|_{B_{1}}\left\|\psi_{i}\right\|_{B_{2}}$ over all representations of $f=\sum_{i \in \mathcal{I}} \phi_{i} \otimes \psi_{i}$.

The following identification of the projective tensor product of $\mathcal{C}_{o_{\pi_{1}}} L_{w_{1}}^{1}\left(G_{1}\right)$ and $\mathcal{C}_{o_{\pi_{2}}} L_{w_{2}}^{1}\left(G_{2}\right)$ with the coorbit space $\mathcal{C}_{\pi} L_{w}^{1}\left(G_{1} \times G_{2}\right)$ is a generalization of Feichtinger's original result for modulation spaces [13, Theorem $7 \mathrm{D}]$.

Theorem 5. Under the general assumptions on the groups $G_{i}$ and the representations $\left(\pi_{i}, \mathcal{H}_{i}\right)$ we have

$$
\mathcal{C} o_{\pi} L_{w}^{1}\left(G_{1} \times G_{2}\right)=\mathcal{C}_{o_{\pi_{2}}} L_{w_{2}}^{1}\left(G_{2}\right) \widehat{\otimes} \mathcal{C}_{o_{\pi_{1}}} L_{w_{1}}^{1}\left(G_{1}\right) .
$$


Proof. Let $F \in \mathcal{C}_{o_{\pi}} L_{w}^{1}(G)$. Then by Theorem 2 applied to $\pi=\pi_{2} \otimes \pi_{1}, F$ possesses the representation $F=\sum_{i \in \mathcal{I}} \lambda_{i}(F) \pi\left(\gamma_{i}\right) \Psi \in \mathcal{C}_{o_{\pi}} L_{w}^{1}(G)$ with $\gamma_{i}=\left(\gamma_{i, 1}, \gamma_{i, 2}\right) \in$ $G_{1} \times G_{2}$ and $\sum_{i \in \mathcal{I}}\left|\lambda_{i}(F)\right| w\left(\gamma_{i}\right) \leqslant C\|F\|_{\mathcal{C}_{o_{\pi} L_{w}}(G)}$. Using Proposition 1(ii) we obtain that

$$
\begin{aligned}
\sum_{i \in \mathcal{I}} \| & \lambda_{i}(F) \pi_{1}\left(\gamma_{i, 1}\right) \psi_{1}\left\|_{\mathcal{C}_{o_{1}} L_{w_{1}}^{1}\left(G_{1}\right)}\right\| \pi_{2}\left(\gamma_{i, 2}\right) \psi_{2} \|_{\mathcal{C}_{\pi_{2}} L_{w_{2}}^{1}\left(G_{2}\right)} \\
& \leqslant \sum_{i \in \mathcal{I}}\left|\lambda_{i}(F)\right| w_{1}\left(\gamma_{i, 1}\right) w_{2}\left(\gamma_{i, 2}\right)\left\|\psi_{1}\right\|_{\mathcal{C}_{o_{\pi_{1}} L_{w_{1}}}^{1}\left(G_{1}\right)}\left\|\psi_{2}\right\|_{\mathcal{C}_{o_{\pi_{2}} L_{w_{2}}}^{1}\left(G_{2}\right)} \\
& \leqslant C\|F\|_{\mathcal{C}_{o_{\pi}} L_{w}^{1}(G)} .
\end{aligned}
$$

Thus $F \in \mathcal{C} o_{\pi_{2}} L_{w_{2}}^{1}\left(G_{2}\right) \widehat{\otimes} \mathcal{C} o_{\pi_{1}} L_{w_{1}}^{1}\left(G_{1}\right)$, and $\mathcal{C} o_{\pi} L_{w}^{1}(G)$ is continuously embedded into $\mathcal{C}_{\pi_{2}} L_{w_{2}}^{1}\left(G_{2}\right) \widehat{\otimes} \mathcal{C}_{\pi_{\pi_{1}}} L_{w_{1}}^{1}\left(G_{1}\right)$.

Conversely, let $F \in \mathcal{C}_{o_{\pi_{2}}} L_{w_{2}}^{1}\left(G_{2}\right) \widehat{\otimes} \mathcal{C}_{o_{\pi_{1}}} L_{w_{1}}^{1}\left(G_{1}\right)$. Choose a representation $F=\sum_{i \in \mathcal{I}} f_{i, 2} \otimes f_{i, 1}$ with $\sum_{i \in \mathcal{I}}\left\|f_{i, 1}\right\|_{\mathcal{C}_{o_{\pi_{1}} L_{w_{1}}}^{1}\left(G_{1}\right)}\left\|f_{i, 2}\right\|_{\mathcal{C}_{\pi_{\pi_{2}} L_{w_{2}}}\left(G_{2}\right)}<\infty$. Using Fubini's theorem and Proposition 1(ii) yields

$$
\begin{aligned}
\|F\|_{\mathcal{C}_{\pi} L_{w}^{1}(G)} & =\int_{G}\left|V_{\Psi} F(g)\right| w(g) d g \\
& \leqslant \sum_{i \in \mathcal{I}}\left(\int_{G_{1}}\left|V_{\psi_{1}} f_{i, 1}\left(g_{1}\right)\right| w_{1}\left(g_{1}\right) d g_{1}\right) \cdot\left(\int_{G_{2}}\left|V_{\psi_{2}} f_{i, 2}\left(g_{2}\right)\right| w_{2}\left(g_{2}\right) d g_{2}\right) \\
& =\sum_{i \in \mathcal{I}}\left\|f_{i, 1}\right\|_{\mathcal{C}_{\pi_{1}} L_{w_{1}}^{1}\left(G_{1}\right)}\left\|f_{i, 2}\right\|_{\mathcal{C}_{o_{\pi_{2}} L_{w_{2}}}^{1}\left(G_{2}\right)}<\infty .
\end{aligned}
$$

Thus, $\mathcal{C}_{\pi_{\pi_{2}}} L_{w_{2}}^{1}\left(G_{2}\right) \widehat{\otimes} \mathcal{C}_{\pi_{1}} L_{w_{1}}^{1}\left(G_{1}\right) \subseteq \mathcal{C}_{o_{\pi}} L_{w}^{1}(G)$. The equivalence of the norms follows from the inverse mapping theorem.

Once the kernel theorem provides a general description of operators between test functions and distributions, we may try to characterize certain classes of operators by properties of their kernel. Since on the level of the generalized wavelet transform such operators correspond to integral operators (see diagram in Figure 1), we may translate the various versions of Schur's test to kernel theorems for operators between coorbit spaces. Following the procedure in [8, Theorem 3.3], we first formulate a general version of Schur's test and then derive the abstract kernel theorem.

We introduce two classes of mixed norm spaces. For two $\sigma$-finite measure spaces $(X, \mu)$ and $(Y, \nu), 1 \leqslant p \leqslant \infty$, and $m: X \times Y \rightarrow \mathbb{R}_{+}$, we define the spaces $L_{m}^{p, \infty}(X \times Y)$ and $\mathcal{L}_{m}^{p, \infty}(X \times Y)$ by the norms

$$
\|F\|_{L_{m}^{p, \infty}(X \times Y)}:=\underset{y \in Y}{\operatorname{ess} \sup }\left(\int_{X}|F(x, y)|^{p} m(x, y)^{p} d \mu(x)\right)^{1 / p}
$$

and

$$
\|F\|_{\mathcal{L}_{m}^{p, \infty}(X \times Y)}:=\operatorname{ess~sup}_{x \in X}\left(\int_{Y}|F(x, y)|^{p} m(x, y)^{p} d \nu(y)\right)^{1 / p} .
$$

The following version of Schur's test is folklore and can be found in [33, Propositions 5.2 and 5.4] or [27. 
Proposition 6. Let $(X, \mu)$ and $(Y, \nu)$ be $\sigma$-finite measure spaces, let $1 \leqslant p \leqslant \infty$, let $\frac{1}{p}+\frac{1}{q}=1$, and let $T$ be the integral operator $T f(y)=\int_{X} f(x) k_{T}(x, y) d \mu(x)$ with kernel $k_{T}: X \times Y \rightarrow \mathbb{C}$.

(i) The operator $T$ is bounded from $L_{m_{1}}^{1}(X)$ to $L_{m_{2}}^{p}(Y)$ if and only if $k_{T} \in$ $\mathcal{L}_{m_{1}^{-1} \otimes m_{2}}^{p, \infty}(X \times Y)$. In that case

$$
\|T\|_{L_{m_{1}}^{1}(X) \rightarrow L_{m_{2}}^{p}(Y)}=\left\|k_{T}\right\|_{\mathcal{L}_{m_{1}^{-1} \otimes m_{2}}^{p, \infty}}(X \times Y) .
$$

(ii) The operator $T$ is bounded from $L_{m_{1}}^{p}(X)$ to $L_{m_{2}}^{\infty}(Y)$ if and only if $k_{T} \in$ $L_{m_{1}^{-1} \otimes m_{2}}^{q, \infty}(X \times Y)$. In this case

$$
\|T\|_{L_{m_{1}}^{p}(X) \rightarrow L_{m_{2}}^{\infty}(Y)}=\left\|k_{T}\right\|_{L_{m_{1}^{q} \otimes m_{2}}^{q, \infty}}(X \times Y) .
$$

We now characterize the boundedness of operators between certain coorbit spaces.

Theorem 7. Let $1 \leqslant p, q \leqslant \infty$ with $\frac{1}{p}+\frac{1}{q}=1$, and let $m_{j}$ be $w_{j}$-moderate weights on $G_{j}$. If $A$ is a bounded operator from $\mathcal{C}_{\pi_{1}} L_{w_{1}}^{1}\left(G_{1}\right)$ to $\mathcal{C}_{o_{\pi_{2}}} L_{1 / w_{2}}^{\infty}\left(G_{2}\right)$ with kernel $K$, then the following hold:

(i) $A$ is bounded from $\mathcal{C}_{o_{\pi_{1}}} L_{m_{1}}^{1}\left(G_{1}\right)$ to $\mathcal{C}_{o_{\pi_{2}}} L_{m_{2}}^{p}\left(G_{2}\right)$ if and only if its kernel $K$ is in $\mathcal{C}_{\pi} \mathcal{L}_{m_{1}^{-1} \otimes m_{2}}^{p, \infty}\left(G_{1} \times G_{2}\right)$. Its operator norm satisfies

$$
\|A\|_{O p} \asymp\|K\|_{\mathcal{C o}_{\pi} \mathcal{L}_{m_{1}^{-1} \otimes m_{2}}^{p, \infty}(G)} .
$$

(ii) $A$ is bounded from $\mathcal{C}_{\pi_{1}} L_{m_{1}}^{p}\left(G_{1}\right)$ to $\mathcal{C}_{o_{\pi_{2}}} L_{m_{2}}^{\infty}\left(G_{2}\right)$ if and only if its kernel $K$ is in $\mathcal{C}_{\pi} L_{m_{1}^{-1} \times m_{2}}^{q, \infty}\left(G_{1} \times G_{2}\right)$. Its operator norm satisfies

$$
\|A\|_{O p} \asymp\|K\|_{\mathcal{C}_{\pi} L_{m_{1}^{-1} \otimes m_{2}}^{q, \infty}}(G) .
$$

Proof. Since $\mathcal{C}_{\pi_{1}} L_{w_{1}}^{1}\left(G_{1}\right) \subseteq \mathcal{C}_{\pi_{1}} L_{m_{1}}^{1}\left(G_{1}\right)$ and $\mathcal{C}_{\pi_{2}} L_{m_{2}}^{p}\left(G_{2}\right) \subseteq \mathcal{C}_{\pi_{2}} L_{1 / w_{2}}^{\infty}\left(G_{2}\right)$ by (10), the kernel theorem is applicable to the operator $A$, and there exists a kernel $K \in \mathcal{C}_{o_{\pi}} L_{1 / w}^{\infty}\left(G_{1} \otimes G_{2}\right)$ such that

$$
V_{\Psi} K\left(g_{1}, g_{2}\right)=k_{A}\left(g_{1}, g_{2}\right)=\left\langle A \pi_{1}\left(g_{1}\right) \psi_{1}, \pi_{2}\left(g_{2}\right) \psi_{2}\right\rangle .
$$

Assume first that $K \in \mathcal{C} o_{\pi} \mathcal{L}_{m_{1}^{-1} \otimes m_{2}}^{p, \infty}(G)$, which means that $V_{\Psi} K \in \mathcal{L}_{1 / m_{1} \otimes m_{2}}^{p, \infty}(G)$. By Proposition [6, the integral operator $\mathfrak{A}$ defined by the integral kernel $k_{A}$ is bounded from $L_{m_{1}}^{1}\left(G_{1}\right)$ to $L_{m_{2}}^{p}\left(G_{2}\right)$. According to (18) $), A$ factors as $A=V_{\psi_{2}}^{*} \mathfrak{A} V_{\psi_{1}}$, where $V_{\psi_{1}}$ is an isometry from $\mathcal{C}_{\pi_{1}} L_{m_{1}}^{1}\left(G_{1}\right)$ to $L_{m_{1}}^{1}\left(G_{1}\right)$, and $V_{\psi_{2}}^{*}$ is bounded from $L_{m_{2}}^{p}\left(G_{2}\right)$ to $\mathcal{C}_{\pi_{2}} L_{m_{2}}^{p}\left(G_{2}\right)$ by Proposition 1 Consequently $A$ is bounded from $\mathcal{C}_{\pi_{1}} L_{m_{1}}^{1}\left(G_{1}\right)$ to $\mathcal{C}_{\pi_{2}} L_{m_{2}}^{p}\left(G_{2}\right)$. The boundedness estimate follows from

$$
\begin{aligned}
\|A\|_{O p} & \leqslant\left\|V_{\psi_{2}}^{*}\right\|_{O p}\|\mathfrak{A}\|_{L_{m_{1}}^{1}\left(G_{1}\right) \rightarrow L_{m_{2}}^{p}\left(G_{2}\right)}\left\|V_{\psi_{1}}\right\|_{O p} \\
& \leqslant C\left\|k_{A}\right\|_{\mathcal{L}_{m_{1}^{p} \otimes m_{2}}^{p, \infty}(G)}=C\|K\|_{\mathcal{C}_{\pi}} \mathcal{L}_{m_{1}^{p} \otimes m_{2}}^{p, \infty}(G)
\end{aligned}
$$


Conversely, let $A$ be bounded from $\mathcal{C}_{\pi_{1}} L_{m_{1}}^{1}\left(G_{1}\right)$ to $\mathcal{C}_{\pi_{2}} L_{m_{2}}^{p}\left(G_{2}\right)$. Then $A \pi_{1}\left(g_{1}\right) \psi_{1} \in \mathcal{C}_{\pi_{\pi_{2}}} L_{m_{2}}^{p}\left(G_{2}\right)$ and the following estimates make sense:

$$
\begin{aligned}
\|K\|_{\mathcal{C}_{o_{\pi}} \mathcal{L}_{m_{1}^{-1} \otimes m_{2}}^{p, \infty}(G)} & =\left\|V_{\Psi} K\right\|_{\mathcal{L}_{m_{1}^{-1} \otimes m_{2}}^{p, \infty}(G)}=\left\|k_{A}\right\|_{\mathcal{L}_{m_{1}^{-1} \otimes m_{2}}^{p, \infty}}(G) \\
& =\sup _{g_{1} \in G_{1}}\left(\int_{G_{2}}\left|\left\langle A \pi_{1}\left(g_{1}\right) \psi_{1}, \pi_{2}\left(g_{2}\right) \psi_{2}\right\rangle m_{2}\left(g_{2}\right)\right|^{p} d g_{2}\right)^{1 / p} m_{1}\left(g_{1}\right)^{-1} \\
& =\sup _{g_{1} \in G_{1}}\left\|A \pi_{1}\left(g_{1}\right) \psi_{1}\right\|_{\mathcal{C}_{\pi_{2}} L_{m_{2}}^{p}\left(G_{2}\right)} m_{1}\left(g_{1}\right)^{-1} \\
& \leqslant\|A\|_{O p} \sup _{g_{1} \in G_{1}}\left\|\pi_{1}\left(g_{1}\right) \psi_{1}\right\|_{\mathcal{C}_{\pi_{1}} L_{m_{1}}^{1}\left(G_{1}\right)} m_{1}\left(g_{1}\right)^{-1}
\end{aligned}
$$

Since $V_{\psi_{1}} \psi_{1} \in L_{w_{1}}^{1}\left(G_{1}\right)$ and $m_{1}$ is $w_{1}$-moderate and thus satisfies $m_{1}\left(g_{1} h\right) m_{1}\left(g_{1}\right)^{-1}$ $\leqslant w_{1}(h)$, the last expression is bounded by

$$
\begin{gathered}
\sup _{g_{1} \in G_{1}}\left\|\pi_{1}\left(g_{1}\right) \psi_{1}\right\|_{\mathcal{C}_{o_{1}} L_{m_{1}}^{1}\left(G_{1}\right)} \cdot m_{1}\left(g_{1}\right)^{-1}=\sup _{g_{1} \in G_{1}} \int_{G_{1}}\left|\left\langle\psi_{1}, \pi_{1}\left(g_{1}^{-1} h\right) \psi_{1}\right\rangle\right| \frac{m_{1}(h)}{m_{1}\left(g_{1}\right)} d h \\
=\sup _{g_{1} \in G_{1}} \int_{G_{1}} \mid\left\langle\psi_{1}, \pi_{1}(h) \psi_{1}\right\rangle \frac{m_{1}\left(g_{1} h\right)}{m_{1}\left(g_{1}\right)} d h \leqslant\left\|V_{\psi_{1}} \psi_{1}\right\|_{L_{w_{1}}^{1}\left(G_{1}\right)} .
\end{gathered}
$$

Thus $K \in \mathcal{C}_{o_{\pi}} \mathcal{L}_{m_{1}^{-1} \otimes m_{2}}^{p, \infty}(G)$.

Part (ii) follows by using Proposition 6(ii) instead of (i) and is proved similarly.

The following diagram (Figure 1) shows the connection between the different operators and spaces.

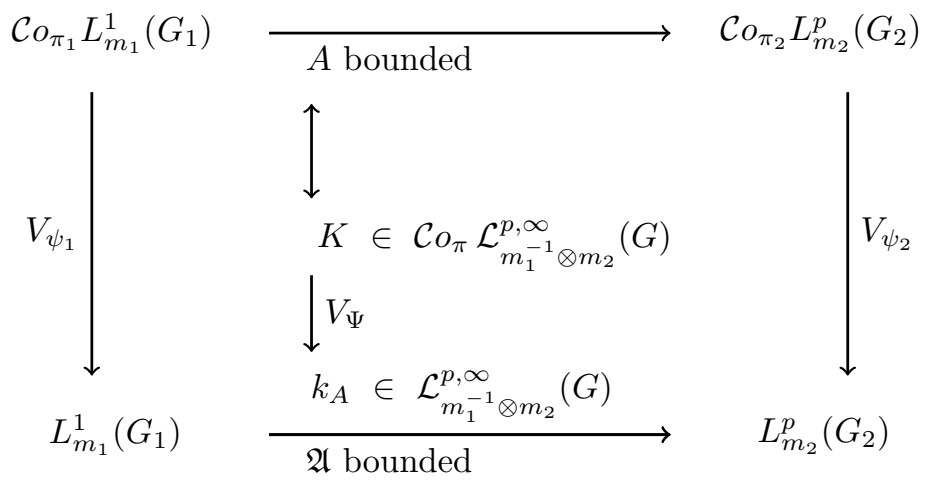

Figure 1

Using interpolation between $L^{p}$-spaces, Schur's test can also be formulated as saying that an integral operator is bounded on all $L^{p}$ simultaneously if and only if its kernel belongs to $L^{1, \infty} \cap \mathcal{L}^{1, \infty}$. The corresponding version for coorbit spaces is a consequence of Theorem 7 and an interpolation argument. 
Corollary 8. The following conditions are equivalent:

(i) $A: \mathcal{C}_{\pi_{1}} L_{m_{1}}^{p}\left(G_{1}\right) \rightarrow \mathcal{C}_{\pi_{2}} L_{m_{2}}^{p}\left(G_{2}\right)$ is bounded for every $1 \leqslant p \leqslant \infty$.

(ii) Both $A: \mathcal{C}_{\pi_{1}} L_{m_{1}}^{1}\left(G_{1}\right) \rightarrow \mathcal{C}_{\pi_{2}} L_{m_{2}}^{1}\left(G_{2}\right)$ and $A: \mathcal{C}_{\pi_{1}} L_{m_{1}}^{\infty}\left(G_{1}\right) \rightarrow$ $\mathcal{C}_{\pi_{2}} L_{m_{2}}^{\infty}\left(G_{2}\right)$ are bounded.

(iii) $K \in \mathcal{C} o_{\pi} \mathcal{L}_{m_{1}^{-1} \otimes m_{2}}^{1, \infty}(G) \bigcap \mathcal{C}_{\pi} L_{m_{1}^{-1} \otimes m_{2}}^{1, \infty}(G)$.

Clearly one can now translate every boundedness result for an integral operator into a kernel theorem for coorbit spaces. As a simple but important example we offer a sufficient condition for regularizing operators, i.e., operators that map distributions to test functions.

Theorem 9. Under the assumptions of Theorem [3, if the unique kernel of the operator $A$ satisfies $K \in \mathcal{C}_{\pi} L_{w}^{1}(G)$, then $A$ is bounded from $\mathcal{C}_{o_{\pi_{1}}} L_{1 / w_{1}}^{\infty}\left(G_{1}\right)$ to $\mathcal{C}_{\pi_{2}} L_{w_{2}}^{1}\left(G_{2}\right)$.

Proof. Consider the integral operator $\mathfrak{A}$ as in the proof of Theorem 7 and observe that $V_{\Psi} K=k_{A} \in L_{w}^{1}(G)$ is a sufficient condition for $\mathfrak{A}: L_{1 / w_{1}}^{\infty}\left(G_{1}\right) \rightarrow L_{w_{2}}^{1}\left(G_{2}\right)$ to be bounded by Schur's test.

4.1. Discretization. Coorbit theory guarantees the discretization of the coorbit spaces via atomic decompositions and Banach frames. For our purposes, it is sufficient to state a shortened and simplified version of [22, Theorem 5.3]. Let $Y$ be one of the function spaces $L_{m}^{p}(G), L_{m}^{p, \infty}(G)$, or $\mathcal{L}_{m}^{p, \infty}(G)$, and let $Y_{d}$ be the natural sequence space associated to $Y$.

Proposition 10. If $\psi$ satisfies

$$
\int_{G} \sup _{h \in g Q}\left|V_{\psi} \psi(h)\right| w(g) d g<\infty,
$$

for a compact neighborhood $Q$ of $e$, then there exist a discrete subset $\Lambda \subset G$ and constants $C_{1}, C_{2}>0$ such that

$$
C_{1}\|f\|_{\mathcal{C}_{\pi} Y} \leqslant\left\|V_{\psi} f\right\|_{Y_{d}} \leqslant C_{2}\|f\|_{\mathcal{C}_{\pi} Y} \quad \text { for every } f \in \mathcal{C}_{o_{\pi}} Y .
$$

Corollary 11. Let $\Lambda=\Lambda_{1} \times \Lambda_{2} \subset G$ be a discrete set such that $\{\pi(\lambda) \Psi\}_{\lambda \in \Lambda}$ satisfies (28) for $\mathcal{C}_{o_{\pi}} L_{m_{1}^{-1} \otimes m_{2}}^{p, \infty}(G)$ and $\mathcal{C}_{\pi} \mathcal{L}_{m_{1}^{-1} \otimes m_{2}}^{p, \infty}(G)$. If $A$ is a bounded operator from $\mathcal{C}_{o_{\pi_{1}}} L_{w_{1}}^{1}\left(G_{1}\right)$ to $\mathcal{C}_{o_{\pi_{2}}} L_{1 / w_{2}}^{\infty}\left(G_{2}\right)$ with kernel $K$, then the following hold:

(i) $A: \mathcal{C}_{\pi_{\pi_{1}}} L_{m_{1}}^{1}\left(G_{1}\right) \rightarrow \mathcal{C}_{o_{\pi_{2}}} L_{m_{2}}^{p}\left(G_{2}\right)$ is bounded if and only if

$$
\sup _{\lambda_{1} \in \Lambda_{1}}\left(\sum_{\lambda_{2} \in \Lambda_{2}}\left|V_{\Psi} K(\lambda)\left(m_{1}^{-1} \otimes m_{2}\right)(\lambda)\right|^{p}\right)^{1 / p}<\infty .
$$

(ii) Likewise $A: \mathcal{C}_{\pi_{1}} L_{m_{1}}^{p}\left(G_{1}\right) \rightarrow \mathcal{C} o_{\pi_{2}} L_{m_{2}}^{\infty}\left(G_{2}\right)$ is bounded if and only if

$$
\sup _{\lambda_{2} \in \Lambda_{2}}\left(\sum_{\lambda_{1} \in \Lambda_{1}}\left|V_{\Psi} K(\lambda)\left(m_{1}^{-1} \otimes m_{2}\right)(\lambda)\right|^{q}\right)^{1 / q}<\infty .
$$

Proof. (i) By Theorem $7 A$ has a kernel in $\mathcal{C}_{\pi} \mathcal{L}^{p, \infty}(G)$, and

$$
\|A\|_{O p} \asymp\|K\|_{\mathcal{C}_{\pi} \mathcal{L}^{p, \infty}(G)} \text {. }
$$

By (28), the expression in (29) is an equivalent norm for $\|K\|_{\mathcal{C}_{\pi} \mathcal{L}^{p, \infty}(G)}$. The proof of (ii) works in exactly the same way. 


\section{EXAMPLES}

5.1. Modulation spaces. The Weyl-Heisenberg group $G_{W H}=\mathbb{R}^{d} \times \mathbb{R}^{d} \times \mathbb{T}$ is defined by the group law

$$
\left(x, \omega, e^{2 \pi i \tau}\right) \cdot\left(x^{\prime}, \omega^{\prime}, e^{2 \pi i \tau^{\prime}}\right)=\left(x+x^{\prime}, \omega+\omega^{\prime}, e^{2 \pi i\left(\tau+\tau^{\prime}-x \cdot \omega^{\prime}\right)}\right) .
$$

Let $T_{x} f(t):=f(t-x)$ denote the translation, and let $M_{\omega} f(t):=e^{2 \pi i \omega t} f(t)$ be the modulation operator. The operator $\pi_{W H}(x, \omega, \tau)=e^{2 \pi i \tau} M_{\omega} T_{x}$ for $(x, \omega, \tau) \in G_{W H}$ defines a unitary square-integrable representation of $G_{W H}$ acting on $L^{2}\left(\mathbb{R}^{d}\right)$, for which every non-zero vector in $L^{2}\left(\mathbb{R}^{d}\right)$ is admissible. Since the phase factor $e^{2 \pi i \tau}$ is irrelevant for the definition of coorbit spaces, it is convenient to drop the trivial third component and consider the time-frequency shift $\pi(x, \omega)=\pi_{W H}(x, \omega, 1)=M_{\omega} T_{x}$. Formally, we treat the projective representation $\pi$ of $\mathbb{R}^{2 d}$ instead of the unitary representation $\pi_{W H}$ of $G_{W H}$. The transform corresponding to $\pi$ is nothing else but the short-time Fourier transform

$$
V_{\psi} f(x, \omega)=\left\langle f, M_{\omega} T_{x} \psi\right\rangle=\int_{\mathbb{R}^{d}} f(t) \overline{\psi(t-x)} e^{-2 \pi i \omega \cdot t} d t, \quad f, \psi \in L^{2}\left(\mathbb{R}^{d}\right) .
$$

The coorbit spaces associated to $\pi_{W H}$ coincide therefore with the coorbit spaces associated to $\pi$. These are the modulation spaces $M_{m}^{p}\left(\mathbb{R}^{d}\right)$ which were first introduced by Feichtinger in [14 as certain decomposition spaces and subsequently were identified with the coorbit spaces of the Heisenberg group $\mathcal{C} o_{\pi_{W H}} L_{m}^{p}\left(G_{W H}\right)=$ $\mathcal{C}_{o_{\pi}} L_{m}^{p}\left(\mathbb{R}^{2 d}\right)=M_{m}^{p}\left(\mathbb{R}^{d}\right)$ [18. We refer to the standard textbooks [20, 23] for more information on time-frequency analysis.

Theorem 3 asserts that every bounded operator from $M_{w}^{1}\left(\mathbb{R}^{d}\right)=\mathcal{C} o_{\pi_{W H}} L_{w}^{1}\left(G_{W H}\right)$ $=\mathcal{C}_{o_{\pi}} L_{w}^{1}\left(\mathbb{R}^{2 d}\right)$ to $M_{1 / w}^{\infty}\left(\mathbb{R}^{d}\right)=\mathcal{C}_{o_{\pi_{W H}}} L_{1 / w}^{\infty}\left(G_{W H}\right)=\mathcal{C} o_{\pi} L_{1 / w}^{\infty}\left(\mathbb{R}^{2 d}\right)$ possesses a kernel $K \in \mathcal{C}_{\pi_{W H} \otimes \pi_{W H}} L_{w^{-1} \otimes w^{-1}}^{\infty}\left(G_{W H} \times G_{W H}\right)$ such that $\langle A f, g\rangle=\langle K, g \otimes f\rangle$ for $f, g \in M_{w}^{1}\left(\mathbb{R}^{d}\right)$. Let us elaborate in detail what the kernel theorem asserts in this case: for $g_{i}=\left(x_{i}, \omega_{i}, \tau_{i}\right) \in G_{W H}, i=1,2$, the tensor representation $\pi_{W H} \otimes \pi_{W H}$ acts on the simple tensor $\left(\psi_{2} \otimes \psi_{1}\right)\left(t_{2}, t_{1}\right)=\psi_{2}\left(t_{2}\right) \overline{\psi_{1}\left(t_{1}\right)} \in L^{2}\left(\mathbb{R}^{d}\right) \otimes L^{2}\left(\mathbb{R}^{d}\right) \cong$ $L^{2}\left(\mathbb{R}^{2 d}\right)$ as

$$
\begin{array}{r}
\pi_{W H} \otimes \pi_{W H}\left(g_{2}, g_{1}\right)\left(\psi_{2} \otimes \psi_{1}\right)\left(t_{2}, t_{1}\right)=e^{2 \pi i\left(\tau_{1}-\tau_{2}\right)} M_{\omega_{2}} T_{x_{2}} \psi_{2}\left(t_{2}\right) \overline{M_{\omega_{1}} T_{x_{1}} \psi_{1}\left(t_{1}\right)} \\
=e^{2 \pi i\left(\tau_{1}-\tau_{2}\right)} M_{\left(\omega_{2},-\omega_{1}\right)} T_{\left(x_{2}, x_{1}\right)}\left(\psi_{2} \otimes \psi_{1}\right)\left(t_{2}, t_{1}\right) .
\end{array}
$$

Thus except for the phase factor $e^{2 \pi i\left(\tau_{1}-\tau_{2}\right)}$ the tensor representation $\pi_{W H} \otimes \pi_{W H}$ is just the time-frequency shift $M_{\left(\omega_{2},-\omega_{1}\right)} T_{\left(x_{2}, x_{1}\right)}$ acting on $L^{2}\left(\mathbb{R}^{2 d}\right)$. Consequently, the coorbit spaces with respect to the product group $G_{W H} \otimes G_{W H}$ are again modulation spaces, this time on $\mathbb{R}^{2 d}$. For the coorbit of $L^{\infty}$ we compare the norms

$$
\|K\|_{M^{\infty}\left(\mathbb{R}^{2 d}\right)}=\sup _{\left(x_{1}, x_{2}, \omega_{1}, \omega_{2}\right) \in \mathbb{R}^{4 d}}\left|\left\langle K, M_{\left(\omega_{1}, \omega_{2}\right)} T_{\left(x_{1}, x_{2}\right)}\left(\psi_{2} \otimes \psi_{1}\right)\right\rangle\right|
$$

and

$$
\|K\|_{\mathcal{C}_{\pi \otimes \pi} L^{\infty}\left(\mathbb{R}^{4 d}\right)}=\sup _{\left(\left(x_{1}, \omega_{1}\right),\left(x_{2}, \omega_{2}\right)\right) \in \mathbb{R}^{4 d}}\left|\left\langle K,\left(\pi\left(x_{1}, \omega_{1}\right) \otimes \pi\left(x_{2}, \omega_{2}\right)\right)\left(\psi_{2} \otimes \psi_{1}\right)\right\rangle\right|,
$$

which are obviously equal. In this case Theorem 3 is therefore just Feichtinger's kernel theorem: For $A: M^{1}\left(\mathbb{R}^{d}\right) \rightarrow M^{\infty}\left(\mathbb{R}^{d}\right)$ there exists a unique kernel $K \in$ $M^{\infty}\left(\mathbb{R}^{2 d}\right)$ such that $\langle A f, g\rangle=\langle K, g \otimes f\rangle$. 
The recent extension of Feichtinger's kernel theorem by Cordero and Nicola 8 can be seen in the same light. Let us explain the difference in the formulations. Our approach considers the generalized wavelet transform

$$
V_{\Psi} K\left(x_{1}, \omega_{1}, x_{2}, \omega_{2}\right)=\left\langle K, \pi_{W H}\left(x_{2}, \omega_{2}, 1\right) \otimes \pi_{W H}\left(x_{1}, \omega_{1}, 1\right)\left(\psi_{2} \otimes \psi_{1}\right)\right\rangle
$$

of the kernel. The conditions of Theorem 7 are formulated by mixed norms acting simultaneously on the variables $\left(x_{2}, \omega_{2}\right)$ and $\left(x_{1}, \omega_{1}\right)$. The treatment in 8 uses the short-time Fourier transform on $\mathbb{R}^{2 d}$

$$
V_{\Psi} K\left(x_{1}, x_{2}, \omega_{1}, \omega_{2}\right)=\left\langle K, M_{\left(\omega_{1}, \omega_{2}\right)} T_{\left(x_{1}, x_{2}\right)} \Psi\right\rangle,
$$

which is the same transform, except for the order of the variables. In [8] it was therefore necessary to reshuffle the order of integration of time-frequency shifts and to use the notion of mixed modulation spaces, which were studied in [4,29. The new insight of our formulation is that the mixed modulation spaces are simply the coorbit spaces with respect to the tensor product representation.

The special case of Theorem 7 for the Weyl-Heisenberg group and the weights $m_{s}(x, \omega, \tau)=(1+|x|+|\omega|)^{s}$ for $s \in \mathbb{R}$ states the following: Fix $\sigma>0$ and let $A$ be an operator from $M_{m_{\sigma}}^{1}\left(\mathbb{R}^{d}\right)$ to $M_{m_{-\sigma}}^{\infty}\left(\mathbb{R}^{d}\right)$. Then for $|r|,|s| \leqslant \sigma, 1 \leqslant p, q \leqslant \infty$, and $1 / p+1 / q=1$ we have

$\begin{array}{lll}\text { (i) } & A: M_{m_{s}}^{1}\left(\mathbb{R}^{d}\right) \rightarrow M_{m_{r}}^{p}\left(\mathbb{R}^{d}\right) \text { bounded } \Leftrightarrow K \in \mathcal{C}_{\pi} \mathcal{L}_{m_{-s} \otimes w_{r}}^{p, \infty}\left(\mathbb{R}^{4 d}\right), \\ \text { (ii) } & A: M_{m_{s}}^{p}\left(\mathbb{R}^{d}\right) \rightarrow M_{m_{r}}^{\infty}\left(\mathbb{R}^{d}\right) \text { bounded } \Leftrightarrow K \in \mathcal{C}_{\pi} L_{m_{-s} \otimes m_{r}}^{q, \infty}\left(\mathbb{R}^{4 d}\right) .\end{array}$

Regularizing operators from $M^{\infty}$ to $M^{1}$ were recently studied by Feichtinger and Jakobsen [19]: they characterized a subclass of this space of operators by an integral kernel in $M^{1}\left(\mathbb{R}^{2 d}\right)$. The sufficiency of this result in a coorbit version is contained in Theorem 9

5.2. Wavelet coorbit spaces and Besov spaces. The affine group $G_{\text {aff }}=\mathbb{R} \times \mathbb{R}^{*}$ is given by the group law $(x, a) \cdot(y, b)=(x+a y, a b)$, where $x, y \in \mathbb{R}$ and $a, b \in \mathbb{R} \backslash\{0\}$. Its left Haar measure is given by $\frac{d x d a}{a^{2}}$. Let $D_{a} f(t)=|a|^{-1 / 2} f(t / a)$ denote the dilation operator. Then $(x, a) \rightarrow \pi_{\text {aff }}(x, a)=T_{x} D_{a}$ defines a unitary, squareintegrable representation of $G_{\text {aff }}$ on $L^{2}(\mathbb{R})$.

Now let $f, \psi \in L^{2}(\mathbb{R})$. The continuous wavelet transform is defined as

$$
W_{\psi} f(x, a):=\left\langle f, \pi_{\mathrm{aff}}(x, a) \psi\right\rangle=|a|^{-1 / 2} \int_{\mathbb{R}} f(t) \overline{\psi\left(a^{-1}(t-x)\right)} d t,
$$

and the admissibility condition (3) reads as

$$
\int_{\mathbb{R}^{*}}|\widehat{\psi}(\omega)|^{2} \frac{d \omega}{|\omega|}<\infty
$$

It is well-known that the coorbit spaces associated to the representation $\pi_{\text {aff }}$ are the homogeneous Besov spaces. See the textbooks [10, 28] for details and further expositions of wavelet theory. For brevity, we consider only the coorbit spaces with respect to the weighted $L^{p}\left(G_{\text {aff }}\right)$-spaces with the weight function $\nu_{s}(x, a)=\nu_{s}(a)=|a|^{-s}$ for $s \in \mathbb{R}$. Note that $\nu_{-s}=1 / \nu_{s}$. Then $\mathcal{C}_{\pi_{\pi_{\mathrm{aff}}}} L_{\nu_{s}}^{p}\left(G_{\mathrm{aff}}\right)=$ $\dot{B}_{p, p}^{s-1 / 2+1 / p}(\mathbb{R})$ by [15, Section 7.2]. In particular $\mathcal{C}_{\pi_{\text {aff }}} L_{\nu_{s}}^{1}\left(G_{a}\right)=\dot{B}_{1,1}^{s+1 / 2}(\mathbb{R})$ and $\mathcal{C} o_{\pi_{\text {aff }}} L_{\nu_{s}}^{\infty}\left(G_{\text {aff }}\right)=\dot{B}_{\infty, \infty}^{s-1 / 2}(\mathbb{R})$. In this example Theorem 3 states that an operator $A: \dot{B}_{1,1}^{s}(\mathbb{R}) \rightarrow \dot{B}_{\infty, \infty}^{-r}(\mathbb{R})$ is bounded if and only if its associated kernel $K$ is in $\mathcal{C}_{\pi_{\text {aff }} \otimes \pi_{\text {aff }}} L_{\nu_{-s-1 / 2} \otimes \nu_{-r-1 / 2}}^{\infty}\left(G_{\text {aff }}^{2}\right)$. At first glance not much seems to have been 
gained by this formulation, but it turns out that the coorbit spaces of the tensor product $\pi_{\text {aff }} \otimes \pi_{\text {aff }}$ of $G_{\text {aff }}^{2}$ are well understood in the theory of function spaces under the name of Besov spaces of dominating mixed smoothness. In particular, $\mathcal{C} O_{\pi_{\text {aff }} \otimes \pi_{\text {aff }}} L_{\nu_{-s-1 / 2} \otimes \nu_{-r-1 / 2}}^{\infty}\left(G_{\text {aff }}^{2}\right)$ can be identified with the Besov space of dominating mixed smoothness $S_{\infty, \infty}^{-s,-r} B\left(\mathbb{R}^{2}\right)$. See [32, Definition A.4] and 31]. Moreover, Theorem 7 yields a characterization of continuous operators between certain Besov spaces:

(i) $A: \dot{B}_{1,1}^{s}(\mathbb{R}) \rightarrow \dot{B}_{p, p}^{r}(\mathbb{R}) \quad$ bounded $\Leftrightarrow K \in \mathcal{C} o_{\pi_{\text {aff }} \otimes \pi_{\text {aff }}} \mathcal{L}_{\nu_{-s+1 / 2} \otimes \nu_{r+1 / 2-1 / p}}^{p, \infty}\left(G_{\text {aff }}^{2}\right)$,

(ii) $A: \dot{B}_{p, p}^{s}(\mathbb{R}) \rightarrow \dot{B}_{\infty, \infty}^{r}(\mathbb{R})$ bounded $\Leftrightarrow K \in \mathcal{C} o_{\pi_{\mathrm{aff}} \otimes \pi_{\mathrm{aff}}} L_{\nu_{-s-1 / 2+1 / p} \otimes \nu_{r+1 / 2}}^{q, \infty}\left(G_{\mathrm{aff}}^{2}\right)$.

The case (i) for $p=1$ was already formulated in a discrete version by Meyer [28, Section 6.9, Proposition 6].

Theorem 12. Let $\left\{\psi_{k, j}\right\}_{(k, j) \in \mathbb{Z}^{2}}$ be a wavelet basis with $\psi_{k, j}(t)=2^{j / 2} \psi\left(2^{j} t-k\right)$, and assume that $\psi$ has compact support and satisfies sufficiently many moment conditions so that the assumption of Proposition 10 is satisfied. An operator $A$ : $\dot{B}_{1,1}^{0}(\mathbb{R}) \rightarrow \dot{B}_{1,1}^{0}(\mathbb{R})$ is bounded if and only if

$$
\sup _{(k \prime, j \prime) \in \mathbb{Z}^{2}} \sum_{(k, j) \in \mathbb{Z}^{2}}\left|\left\langle A \psi_{k \prime, j \prime}, \psi_{k, j}\right\rangle\right| 2^{-j / 2+j^{\prime} / 2} \leqslant C .
$$

Proof. Set $p=1, s=-1 / 2$, recall that $k_{A}=V_{\Psi} K$, and apply Corollary 11

5.3. The case of two distinct representations. For most applications it suffices to consider a single group $G$ and its product group $G \times G$. Our formulation with two different groups allows us to study operators acting between coorbit spaces associated with different group representations. Using the representations of the Weyl-Heisenberg group and the affine group of Sections 5.1 and 5.2, one can characterize the boundedness of operators between certain modulation spaces and Besov spaces by properties of their associated kernels. Theorem 7 now reads as follows:

(i) $A: M_{m_{s}}^{1}\left(\mathbb{R}^{d}\right) \rightarrow \dot{B}_{p, p}^{r}(\mathbb{R})$ bdd.

$$
\Leftrightarrow K \in \mathcal{C}_{\sigma_{\pi_{\mathrm{aff}} \otimes \pi_{W H}} \mathcal{L}_{\widetilde{m}}^{p, \infty}{ }_{-s} \otimes \nu_{r+1 / 2-1 / p}}\left(G_{W H} \times G_{\mathrm{aff}}\right),
$$

(ii) $A: M_{m_{s}}^{p}\left(\mathbb{R}^{d}\right) \rightarrow \dot{B}_{\infty, \infty}^{r}(\mathbb{R})$ bdd.

$$
\Leftrightarrow K \in \mathcal{C} o_{\pi_{\mathrm{aff}} \otimes \pi_{W H}} L_{m_{-s} \otimes \nu_{r+1 / 2}}^{q, \infty}\left(G_{W H} \times G_{\mathrm{aff}}\right),
$$

(iii) $A: \dot{B}_{1,1}^{r}(\mathbb{R}) \rightarrow M_{m_{s}}^{p}\left(\mathbb{R}^{d}\right)$ bdd.

$$
\Leftrightarrow K \in \mathcal{C}_{o_{W H} \otimes \pi_{\mathrm{aff}}} \mathcal{L}_{m_{-r+1 / 2} \otimes \nu_{s}}^{p, \infty}\left(G_{a} \times G_{W H}\right),
$$

(iv) $A: \dot{B}_{p, p}^{r}(\mathbb{R}) \rightarrow M_{m_{s}}^{\infty}\left(\mathbb{R}^{d}\right)$ bdd.

$$
\Leftrightarrow K \in \mathcal{C} o_{\pi_{\mathrm{WH}} \otimes \pi_{\mathrm{aff}}} L_{\nu_{-r-1 / 2+1 / p} \otimes m_{s}}^{q, \infty}\left(G_{a} \times G_{W H}\right) .
$$

As a special case one obtains a characterization of the bounded operators $A$ from $\dot{B}_{1,1}^{r}(\mathbb{R})$ to $L^{2}\left(\mathbb{R}^{d}\right)$. Since $M^{2}\left(\mathbb{R}^{d}\right)=L^{2}\left(\mathbb{R}^{d}\right)$, they are completely characterized by the membership of their kernel in $\mathcal{C}_{\pi_{\text {aff }} \otimes \pi_{W H}} \mathcal{L}_{1 \otimes m_{-r+1 / 2}}^{2, \infty}\left(G_{\text {aff }} \times G_{W H}\right)$.

\section{REFERENCES}

[1] S. T. Ali, J.-P. Antoine, and J.-P. Gazeau, Square integrability of group representations on homogeneous spaces. I. Reproducing triples and frames (English, with French summary), Ann. Inst. H. Poincaré Phys. Théor. 55 (1991), no. 4, 829-855. MR1144104 
[2] Peter Balazs, Matrix representation of operators using frames, Sampl. Theory Signal Image Process. 7 (2008), no. 1, 39-54. MR2455829

[3] P. Balazs and K. Gröchenig. A guide to localized frames and applications to Galerkin-like representations of operators. In I. Pesenson, H. Mhaskar, A. Mayeli, Q. T. L. Gia, and D.X. Zhou, editors, Frames and Other Bases in Abstract and Function Spaces, Applied and Numerical Harmonic Analysis series (ANHA). Birkhauser/Springer, 2017. MR3700112

[4] Shannon Bishop, Mixed modulation spaces and their application to pseudodifferential operators, J. Math. Anal. Appl. 363 (2010), no. 1, 255-264, DOI 10.1016/j.jmaa.2009.08.032. MR2559061

[5] Marcin Bownik, Lyapunov's theorem for continuous frames, Proc. Amer. Math. Soc. 146 (2018), no. 9, 3825-3838, DOI 10.1090/proc/14088. MR 3825837

[6] Jens Gerlach Christensen and Gestur Ólafsson, Coorbit spaces for dual pairs, Appl. Comput. Harmon. Anal. 31 (2011), no. 2, 303-324, DOI 10.1016/j.acha.2011.01.004. MR2806486

[7] John B. Conway, A course in functional analysis, 2nd ed., Graduate Texts in Mathematics, vol. 96, Springer-Verlag, New York, 1990. MR1070713

[8] Elena Cordero and Fabio Nicola, Kernel theorems for modulation spaces, J. Fourier Anal. Appl. 25 (2019), no. 1, 131-144, DOI 10.1007/s00041-017-9573-3. MR3901921

[9] S. Dahlke, F. De Mari, E. De Vito, D. Labate, G. Steidl, G. Teschke, and S. Vigogna, Coorbit spaces with voice in a Fréchet space, J. Fourier Anal. Appl. 23 (2017), no. 1, 141-206, DOI 10.1007/s00041-016-9466-x. MR3602813

[10] Ingrid Daubechies, Ten lectures on wavelets, CBMS-NSF Regional Conference Series in Applied Mathematics, vol. 61, Society for Industrial and Applied Mathematics (SIAM), Philadelphia, PA, 1992. MR.1162107

[11] M. Duflo and Calvin C. Moore, On the regular representation of a nonunimodular locally compact group, J. Functional Analysis 21 (1976), no. 2, 209-243, DOI 10.1016/00221236(76)90079-3. MR0393335

[12] Hans G. Feichtinger, Un espace de Banach de distributions tempérées sur les groupes localement compacts abéliens (French, with English summary), C. R. Acad. Sci. Paris Sér. A-B 290 (1980), no. 17, A791-A794. MR580567

[13] Hans G. Feichtinger, On a new Segal algebra, Monatsh. Math. 92 (1981), no. 4, 269-289, DOI 10.1007/BF01320058. MR643206

[14] H. G. Feichtinger. Modulation spaces on locally compact abelian groups. In Proceedings of "International Conference on Wavelets and Applications" 2002, pages 99-140, Chennai, India, 2003. Updated version of a technical report, University of Vienna, 1983.

[15] Hans G. Feichtinger and Karlheinz Gröchenig, A unified approach to atomic decompositions via integrable group representations, Function spaces and applications (Lund, 1986), Lecture Notes in Math., vol. 1302, Springer, Berlin, 1988, pp. 52-73, DOI 10.1007/BFb0078863. MR 942257

[16] Hans G. Feichtinger and K. H. Gröchenig, Banach spaces related to integrable group representations and their atomic decompositions. I, J. Funct. Anal. 86 (1989), no. 2, 307-340, DOI 10.1016/0022-1236(89)90055-4. MR.1021139

[17] Hans G. Feichtinger and K. H. Gröchenig, Banach spaces related to integrable group representations and their atomic decompositions. II, Monatsh. Math. 108 (1989), no. 2-3, 129-148, DOI 10.1007/BF01308667. MR:1026614

[18] Hans G. Feichtinger and Karlheinz Gröchenig, Gabor wavelets and the Heisenberg group: Gabor expansions and short time Fourier transform from the group theoretical point of view, Wavelets, Wavelet Anal. Appl., vol. 2, Academic Press, Boston, MA, 1992, pp. 359-397. MR.1161258

[19] H. G. Feichtinger and M. S. Jakobsen, The inner kernel theorem for a certain Segal algebra, arXiv:1806.06307, 2018.

[20] Gerald B. Folland, Harmonic analysis in phase space, Annals of Mathematics Studies, vol. 122, Princeton University Press, Princeton, NJ, 1989. MR983366

[21] I. M. Gel'fand and G. E. Shilov, Generalized functions. Vol. 2, Spaces of fundamental and generalized functions; Translated from the 1958 Russian original [ MR0106409] by Morris D. Friedman, Amiel Feinstein and Christian P. Peltzer; Reprint of the 1968 English translation [ MR0230128], AMS Chelsea Publishing, Providence, RI, 2016. MR3469849

[22] Karlheinz Gröchenig, Describing functions: atomic decompositions versus frames, Monatsh. Math. 112 (1991), no. 1, 1-42, DOI 10.1007/BF01321715. MR.1122103 
[23] Karlheinz Gröchenig, Foundations of time-frequency analysis, Applied and Numerical Harmonic Analysis, Birkhäuser Boston, Inc., Boston, MA, 2001. MR1843717

[24] Lars Hörmander, The analysis of linear partial differential operators. I, Distribution theory and Fourier analysis, 2nd ed., Springer Study Edition, Springer-Verlag, Berlin, 1990. MR.1065136

[25] Richard V. Kadison and John R. Ringrose, Fundamentals of the theory of operator algebras. Vol. I, Elementary theory, reprint of the 1983 original, Graduate Studies in Mathematics, vol. 15, American Mathematical Society, Providence, RI, 1997. MR.1468229

[26] Viktor Losert, A characterization of the minimal strongly character invariant Segal algebra, Ann. Inst. Fourier (Grenoble) 30 (1980), no. 3, 129-139. MR.597020

[27] Ivor J. Maddox, Infinite matrices of operators, Lecture Notes in Mathematics, vol. 786, Springer, Berlin, 1980. MR568707

[28] Yves Meyer, Wavelets and operators, translated from the 1990 French original by D. H. Salinger, Cambridge Studies in Advanced Mathematics, vol. 37, Cambridge University Press, Cambridge, 1992. MR1228209

[29] Shahla Molahajloo, Kasso A. Okoudjou, and Götz E. Pfander, Boundedness of multilinear pseudo-differential operators on modulation spaces, J. Fourier Anal. Appl. 22 (2016), no. 6, 1381-1415, DOI 10.1007/s00041-016-9461-2. MR.3572906

[30] A. Perelomov, Generalized coherent states and their applications, Texts and Monographs in Physics, Springer-Verlag, Berlin, 1986. MR858831

[31] H.-J. Schmeisser and H. Triebel, Topics in Fourier analysis and function spaces, Mathematik und ihre Anwendungen in Physik und Technik [Mathematics and its Applications in Physics and Technology], vol. 42, Akademische Verlagsgesellschaft Geest \& Portig K.-G., Leipzig, 1987. MR900143

[32] Winfried Sickel and Tino Ullrich, Tensor products of Sobolev-Besov spaces and applications to approximation from the hyperbolic cross, J. Approx. Theory 161 (2009), no. 2, 748-786, DOI 10.1016/j.jat.2009.01.001. MR.2563079

[33] T. Tao, Lecture Notes 2 for 247 A: Fourier Analysis, www.math.ucla.edu/ tao/247a.1.06f/ notes2.pdf.

[34] Ernest B. Vinberg, Linear representations of groups, translated from the 1985 Russian original by A. Iacob, reprint of the 1989 translation, Modern Birkhäuser Classics, Birkhäuser/Springer, New York, 2010. MR2761806

Acoustics Research Institute, Austrian Academy of Sciences, Wohllebengasse 12-14, 1040 Vienna, Austria

Email address: peter.balazs@oeaw.ac.at

Faculty of Mathematics, University of Vienna, Oskar-Morgenstern-Platz 1, A-1090 Vienna, Austria

Email address: karlheinz.groechenig@univie.ac.at

Institut de Mathématiques de Bordeaux, Université de Bordeaux, 351, Cours de la LibÉration - F 33405 TALEnCe, France

Email address: speckbacher@kfs. oeaw.ac.at 